ARTAZA, Osvaldo. "Sistemas de prevención de delitos o programas de cumplimiento. Breve descripción de las reglas técnicas de gestión del riesgo empresarial y su utilidad en sede jurídico penal"

Polít. crim. Vol. 8, № 16 (Diciembre 2013), Art. 6, pp. 544 - 573. [http://www.politicacriminal.cl/Vol_08/n_16/Vol8N16A6.pdf]

\title{
Sistemas de prevención de delitos o programas de cumplimiento. Breve descripción de las reglas técnicas de gestión del riesgo empresarial y su utilidad en sede jurídico penal.
}

\author{
Osvaldo Artaza Varela \\ Licenciado en Ciencias Jurídicas y Sociales, Universidad de Chile \\ Magister en Derecho Penal y Procesal Penal, Universidad Alberto Hurtado \\ Doctor en Derecho y Ciencias Políticas, Universidad de Barcelona \\ Osvaldo.artaza@gmail.com
}

\section{Resumen}

El presente artículo tiene por objeto analizar de una serie de reglas técnicas destinadas a la gestión del riesgo en el marco de la actividad de la empresa y que son de suma utilidad para la determinación de las expectativas que recaen sobre las personas jurídicas relativas a la prevención de las conductas delictivas que pueden derivar de su propia actividad. Como se defenderá en el presente trabajo, son estas reglas técnicas las que dotarán de contenido a los modelos de prevención de delitos o Programas de cumplimiento que deben implementar las empresas, de acuerdo a lo dispuesto por el artículo $4^{\circ}$ de la Ley 20.393.

Palabras clave: Sistema de prevención de delitos, programa de cumplimiento, gestión de riesgos, ambiente de control, denuncias, evaluación de riesgos, cultura organizacional.

\begin{abstract}
This article proposes to introduce the analysis of a set of technical rules designed for risk management in the context of the company's business, and that are extremely useful for determining the expectations on corporations regarding the prevention of criminal behavior that may result from their own activity. As defended in this paper, these technical rules will provide the necessary content of crime prevention models or the Compliance Programs that companies must implement according to Article 4 of Law 20.393.
\end{abstract}

Key words: Crime prevention system, compliance program, risk management, internal control environment, complaints, risk assessment, organizational culture.

\section{Introducción.}

Una vez que se ha incorporado al ordenamiento jurídico la responsabilidad penal de las empresas, uno de los problemas más interesantes que surgen es el de determinar las posibilidades de respuesta que puede dar la organización frente a la imputación. Como 
ARTAZA, Osvaldo. "Sistemas de prevención de delitos o programas de cumplimiento. Breve descripción de las reglas técnicas de gestión del riesgo empresarial y su utilidad en sede jurídico penal"

punto de partida se podría decir sin problema alguno que, en nuestro caso, esta posibilidad de respuesta está contenida en el artículo $3^{\circ}$ de la Ley $20.393 .^{1}$

Este cuerpo legal introduce a nuestro ordenamiento jurídico la imposición de una verdadera pena para la empresa. En conformidad a las exigencias impuestas por el principio de culpabilidad, para la aplicación de esta medida no basta que un integrante de la empresa haya cometido alguno de los delitos por los cuales se permite la sanción a la empresa, ni que lo haya hecho en beneficio o en nombre de la misma o actuando en representación de ésta. ${ }^{2}$ Es necesario, en todo caso, un hecho propio que pueda atribuirse al sujeto de la imputación. En suma, la responsabilidad por el hecho ajeno y la responsabilidad objetiva se encuentran prohibidas. ${ }^{3}$

Dentro de las posibilidades que tiene la empresa para defenderse de la imputación, de responder a la misma, destaca por su importancia el tratamiento que ha tenido el hecho de que con anterioridad a la conducta delictiva en cuestión haya implementado un programa de cumplimiento, sistema o modelo de prevención de delitos. Lo que en pocas palabras puede ser definido como el conjunto de medidas adoptadas por la empresa para controlar dentro de lo exigible los peligros de infracción al ordenamiento jurídico que pueden derivar de su misma actividad.

\footnotetext{
${ }^{1}$ El art. $3^{\text {o }}$ de Ley 20.393 dispone: "Atribución de responsabilidad penal. Las personas jurídicas serán responsables de los delitos señalados en el artículo $1^{\circ}$ que fueren cometidos directamente e inmediatamente en su interés o para su provecho, por sus dueños, controladores, responsables, ejecutivos principales, representantes o quienes realicen actividades de administración y supervisión, siempre que la comisión del delito fuere consecuencia del incumplimiento, por parte de ésta, de los deberes de dirección y supervisión.

Bajo los mismos presupuestos del inciso anterior, serán también responsables las personas jurídicas por los delitos cometidos por personas naturales que estén bajo la dirección o supervisión directa de alguno de los sujetos mencionados en el inciso anterior.

Se considerará que los deberes de dirección y supervisión se han cumplido cuando con anterioridad a la comisión del delito, la persona jurídica hubiere adoptado e implementado modelos de organización, administración y supervisión para prevenir delitos como el cometido, conforme a lo dispuesto en el artículo siguiente". Como se puede observar, en forma expresa se reconoce que la empresa puede responder a la imputación sosteniendo que se han cumplido sus deberes de dirección y supervisión a través de la implementación de un sistema de modelos de organización, administración y supervisión. Esta opción del legislador chileno coincide con la legislación italiana, que en el art. 6.1 del D. Lgs. N. 231/2001 dispone casos en que la empresa no responde de los delitos cometidos por los integrantes de la misma considerados en el art. 5.1 del mismo decreto.

${ }^{2}$ En relación con estos criterios para la atribución de responsabilidad penal a la empresa en la práctica norteamericana, ver ARTAZA VARELA, O., La empresa como sujeto de imputación de responsabilidad penal. Fundamentos y límites, Barcelona: Ed. Marcial Pons, 2013, pp. 89 y ss. Respecto a los criterios de imputación objetivos para la imposición de responsabilidad administrativa directa a la empresa en Italia, ver DE SIMONE, G., "La responsabilità da reato degli enti: natura giuridica e criteri (oggettivi) di imputazione", Diritto Penale Contemporaneo, pp. 22 y ss. http://www.penalecontemporaneo.it/materia/5-/-//1809\%201a responsabilit da reato degli_enti natura giuridica e criteri oggettivi di imputazione/, 20-05-13. Ver para el caso chileno, HERNÁNDEZ BASUALTO, Héctor, "La introducción de la responsabilidad penal de las personas jurídicas en Chile", Política Criminal, Vol. 5, No 9 (Julio 2010), Art. 5, pp. 216 y ss., http://www.politicacriminal.cl/Vol_05/n_09/Vol5N9A5.pdf (última visita 20/05/2013).

${ }^{3}$ En contra NIETO MARTÍN, A., La responsabilidad penal de las personas jurídicas: Un modelo legislativo, Madrid: Ed. Iustel, pp. 102 y ss. Si bien se manifiesta en contra de esta forma de atribución de responsabilidad lo hace en base a criterios muy diversos al de su supuesta ilegitimidad.
} 


\section{Polít. crim. Vol. 8, N 16 (Diciembre 2013), Art. 6, pp. 544 - 573. \\ [http://www.politicacriminal.cl/Vol_08/n_16/Vol8N16A6.pdf]}

Sin embargo, la justificación de tal posibilidad de respuesta está lejos de ser pacífica. Una postura, no poco usual, se centra en las consecuencias negativas que conllevaría sancionar en todo caso a la empresa. Esta aproximación al problema consiste en sostener que si lo que se pretende con la imposición de la pena a la empresa es motivar que la misma controle adecuadamente sus eventuales conductas delictivas, no parecería eficiente sancionarla a todo evento luego de la constatación de que se comete un delito a propósito de su actividad, ya que no existiría incentivo alguno para que adoptaran las medidas necesarias para la prevención de los mismos.

Con todo, tal aproximación no es suficiente si de lo que se trata es de relacionar la implementación de un programa de cumplimiento con la eventual atribución de responsabilidad penal. Y lo anterior se debe a que lo que está en juego es la imposición de una pena $\mathrm{y}$, por lo mismo, se debe atender a una dimensión de legitimidad en su distribución y no exclusivamente a su eventual utilidad. ${ }^{4}$

Acá se partirá de la base que por mandato del principio de culpabilidad, al igual como sucede en el caso de la atribución de responsabilidad penal individual, de las disposiciones jurídicas que permiten la imposición de responsabilidad penal a la empresa emana un verdadero deber, dirigido a la empresa, de prevenir en cierta medida las posibles conductas delictivas que puedan cometerse a propósito de su actividad. ${ }^{5}$ Por lo mismo, el problema más desafiante que se presenta a la hora de interpretar estas mismas, es el de delimitar o dotar de contenido a este deber que se dirige a la organización y que justificaría en caso de inobservancia la atribución de responsabilidad penal.

Esta aproximación es completamente compatible con nuestra legislación. Para comprobar lo anterior se debe tener en cuenta ciertos aspectos básicos de las normas que permiten la imposición de responsabilidad penal a las empresas en Chile. En primer lugar, se debe recordar que ante la situación de ser llamada a responder se otorga a la empresa la posibilidad de sostener que, con independencia de la comisión del delito, se han cumplido por la misma, sus deberes de dirección y supervisión, lo que se constataría, también de acuerdo a la misma ley, ya que la empresa con anterioridad a la comisión del delito ha adoptado e implementado un modelo de organización, administración y supervisión para prevenir delitos como el cometido en el caso en concreto. ${ }^{6}$

\footnotetext{
${ }^{4} \mathrm{Al}$ respecto, fundamental MOLINA FERNÁNDEZ, F., "Presupuestos de la responsabilidad jurídica (análisis de la relación entre libertad y responsabilidad)", ADPCP, Vol. LIII (2000), pp. 187 y ss. Ver también NINO C., Los escritos de Carlos s., en: NINO, Fundamentos de Derecho penal, Argentina: Ed. Gedisa, 2008, pp. 38 y ss.; ALCÁCER GUIRAO, R., "Prevención y Garantías, conflicto y síntesis", DOXA, no 25 (2002), p. 139, según el cual la pena, como institución social se caracterizaría por presentarse como un "arma de doble filo con respecto a la libertad individual: la pena se legitima en función de su labor de protección de la libertad de los ciudadanos, pero para ello se agrede al mismo tiempo, con su imposición, la libertad de los ciudadanos"; SILVA SÁNCHEZ, J.M., Aproximación al Derecho penal contemporáneo, Montevideo: Ed. B de F, 2010 (2 edición), pp. 340 y ss.

${ }^{5}$ Obviamente tal proceso de construcción del deber dependerá de los términos en que se recoja en cada ordenamiento jurídico en particular. Para el caso de la legislación chilena, en donde en forma evidente se puede concluir que emana un deber de evitar resultados delictivos, ver nota 3.

${ }^{6}$ Así mismo, la empresa podría sostener (responder) que el delito fue cometido exclusivamente en ventaja propia o a favor de un tercero. Sin embargo acá no se atenderá a esa posibilidad de respuesta. Ver art. $3^{\circ}$ y $4^{\circ}$
} 
ARTAZA, Osvaldo. "Sistemas de prevención de delitos o programas de cumplimiento. Breve descripción de las reglas técnicas de gestión del riesgo empresarial y su utilidad en sede jurídico penal"

Resulta especialmente relevante la Historia de la Ley 20.393, en donde se refleja la intención del legislador de adoptar un modelo de imputación denominado como "defecto de organización". Este deber se originaría, de acuerdo a esta postura, a partir de un nuevo concepto de culpabilidad que atendería a un sentido más amplio que el "dogmático tradicional" destinado a responder a las exigencias jurídicas que recaen sobre las empresas en la actualidad. El reproche social hacia la empresa se fundamentaría en el fallo organizativo que vulneraría ese deber general que recaería en la empresa de organizarse correctamente.

En forma similar la Fiscalía Nacional, en oficio FN N 440/2010 ha señalado:

“... es posible sostener que la responsabilidad de la empresa no deriva directamente del delito cometido por uno de sus ejecutivos o representantes, sino que es consecuencia del incumplimiento o cumplimiento imperfecto de los deberes de dirección y supervisión de la entidad, que se traducen fundamentalmente en su incapacidad para prevenir su utilización para la comisión de delitos, y precisamente por este defecto en su organización, ha sido posible que uno de sus dueños o ejecutivos haya podido cometer uno de los ilícitos de que trata el artículo $1^{\circ}$ de la ley”.

Si se parte de la base, entonces, que del ordenamiento jurídico-penal se desprende la obligación de controlar ciertos riesgos derivados del propio comportamiento y que pueden afectar a terceros, se debe tener en cuenta, a su vez, que las exigencias de este control no son absolutas, ya que el mismo ordenamiento jurídico puede dar pie a ciertas limitaciones que, en general, vienen dadas por las capacidades del destinatario y qué se espera del mismo, así como por el alcance del contenido de la norma en particular (en este caso, aquella que permite la atribución de responsabilidad penal a la empresa). ${ }^{7}$ Es decir, tales normas que especifican resultados lesivos que deben ser evitados se delimitan no solo en razón de las capacidades de sus destinatarios, sino que también en virtud de su relación con otras normas jurídicas, generando así verdaderas reglas de conducta dirigidas a un destinatario en particular. Especial relevancia reviste para tal definición la norma extrapenal, como también ciertas prácticas asentadas en el sector del que se trate y que se espera que el sujeto que interactúa en el mismo domine como condición al desarrollo de su misma actividad. ${ }^{8}$ El objeto de este artículo es precisamente describir algunas de estas exigencias con el propósito de presentar una primera aproximación acerca de su eventual utilidad para la determinación del alcance de los artículos $3^{\circ}$ y $4^{\circ}$ de la Ley 20.393.

Ley 20.393. Entre nosotros, HERNÁNDEZ, "La introducción de la responsabilidad penal”, cit. nota n 2, pp. 216 y ss.

${ }^{7}$ En detalle, para el caso de la responsabilidad penal individual, KINDHÄUSER, U., "El denominado riesgo no permitido", en: KINDHÄUSER, Urs; POLAINO-ORTS, Miguel; BUOMPADRE, Jorge, Normativismo en Derecho penal. Estudios de dogmática jurídico-penal, Chaco: Ed. ConTexto, 2011, pp. 223 y ss.

${ }^{8}$ Es imposible abordar acá la problemática asociada a la posibilidad de considerar a la empresa como destinataria de normas de conducta. Al respecto SILVA SÁNCHEZ, J.M., Normas y acciones en Derecho Penal, Buenos Aires: Ed. Hammurabi, 2003, pp. 78 y ss. Ver también, aunque en términos muy diversos, ARTAZA, La empresa como sujeto de imputación, cit. nota $\mathrm{n}^{\circ} 2$, pp. 225 y ss. 


\section{Polít. crim. Vol. 8, N 16 (Diciembre 2013), Art. 6, pp. 544 - 573. [http://www.politicacriminal.cl/Vol_08/n_16/Vol8N16A6.pdf]}

Dentro del área que nos ocupa, es decir, la delimitación del deber dirigido a la empresa para que controle los riesgos de comisión de delitos que se generan con su misma actividad son fundamentales, ya que han ido adquiriendo mayor incidencia en la práctica, ciertas reglas técnicas y prácticas asociadas a la gestión de riesgos, que son definidas tanto por el sector público como el sector privado y que se aplican para cualquier organización empresarial como criterios de calidad a la hora de minimizar sus propios riesgos.

Estas prácticas asentadas en el sector empresarial pueden ser de suma utilidad para la delimitación del deber mencionado, ya que derechamente definen el margen de diligencia que debe poner la empresa en el cuidado de los riesgos que genera relativos a la observación del ordenamiento jurídico. ${ }^{9}$

\section{Definición y propósito de los programas de cumplimiento.}

El programa de cumplimiento consiste en el conjunto sistemático de esfuerzos realizados por los integrantes de la empresa tendentes a asegurar que las actividades llevadas a cabo por ésta no vulneren la legislación aplicable. Desde la óptica de la administración empresarial éstos serían un ejemplo de uno de los "sistemas de calidad" que operan en toda actividad empresarial, por lo que contiene aspectos relacionados tanto con la estructura organizacional, distribución de responsabilidades, procedimientos y los recursos utilizados por la empresa para asegurar la calidad de la dirección de ésta. ${ }^{10}$

El primer objeto que se asocia inmediatamente al programa de cumplimiento es el de servir de reunión o sistematización de todas aquellas medidas o procedimientos adoptados por la empresa tendentes a asegurar o promover un comportamiento, por parte de sus integrantes respetuosos con la ley.

Desde la perspectiva que resulta más relevante para la responsabilidad penal de la empresa el programa de cumplimiento debiera tender a disminuir el margen de ocasiones en que la empresa es sancionada penalmente mediante la reducción correlativa de la frecuencia con que se llevan a cabo conductas delictivas en el marco del desarrollo de la actividad empresarial. $^{11}$

\footnotetext{
${ }^{9}$ Solo se pretende entregar algunos ejemplos, en razón de que es absolutamente inabarcable considerar todas estas recomendaciones relativas a la prevención de delitos de la empresa.

${ }^{10}$ GRUNER, R./BROWN, L., "Organizational Justice: Recognizing and Rewarding the Good Citizen Corporation", The Journal of Corporation Law, Vol. 21 (1996), p. 737. Para más definiciones de estos instrumentos, ver WALSH, C.; PYRICH, A., "Corporate Compliance Programs as a Defense to Criminal Liability: Can a Corporation Save its Soul?", Rutgers Law Review, Vol. 47 (1994-1995), pp. 645; WELLNER P., "Effective Compliance Programs and Corporate Criminal Prosecutions", Cardozo Law Review, Vol. 27 (2005), pp. 497 y ss. Ver también, PIÑA ROCHEFORT, Juan Ignacio, Modelos de prevención de delitos en la empresa, Santiago: Ed. Abeledo Perrot/Thomson Reuters, 2012, p. 2, de acuerdo al cual los "modelos de prevención son formas de organización que adopta la persona jurídica con dos objetivos, por una parte, impedir la comisión de delitos y, por la otra, para blindarse del surgimiento de responsabilidad penal por los hechos cometidos por alguno de sus integrantes en caso que ello ocurra".

${ }^{11}$ GRUNER R., Corporate Criminal Liability and Prevention, New York: Ed. Law Journal Press, 2010, pp. $14-4$ y ss.
} 
ARTAZA, Osvaldo. "Sistemas de prevención de delitos o programas de cumplimiento. Breve descripción de las reglas técnicas de gestión del riesgo empresarial

y su utilidad en sede jurídico penal"

Lo anterior se llevaría a cabo por medio de la conducción de las funciones de los integrantes de la empresa a través de un estricto apego a la legalidad, por medio de la generación de incentivos para la adopción de tal forma de comportamiento, así como mediante el establecimiento de un adecuado monitoreo del cumplimiento de éste.

Sin embargo, este conjunto o sistema de medidas adoptados por la empresa, no solo debieran tender a que no se llevara a cabo ningún tipo de conducta delictiva (o un menor número) por parte de los integrantes, sino también a generar la capacidad de detectar oportunamente este tipo de conductas, cuando se encuentren en un nivel de desarrollo irrelevante como para dar pie a una eventual persecución penal en contra de la empresa. Por ejemplo, mediante la detección de procedimientos especialmente riesgosos que pudieran desencadenar ciertos resultados lesivos.

Una de las principales preocupaciones vinculadas a la existencia de un programa de cumplimiento en la empresa estaría dada porque un comportamiento adecuado a las exigencias legales en determinadas áreas relacionadas con la actividad empresarial sería complejo y en muchas ocasiones de difícil determinación.

Como la empresa responde a una serie de reglas de diversa naturaleza para la consecución de sus objetivos (por ejemplo, aquellas que vendrían dadas por la competencia y determinadas prácticas publicitarias necesarias para convencer a los consumidores, o en el caso del mercado financiero la interacción con ciertas prácticas especulativas, etc.) el programa de cumplimiento debiera lograr que en aquellos casos en que la regulación vigente (por ejemplo, la administrativa) resultara de cierta complejidad, se pudiera presentar a los integrantes de la empresa en una forma más accesible a éstos mismos.

Otro papel que se le asigna a éstos es el de compensar cierta orientación a la obtención de beneficios que se manifiestan a través de presiones hacia los integrantes de la empresa, bajo cierta forma de esquemas de incentivos, como sería recurrente, por ejemplo, en aquel sector de la administración a los cuales se les exige que consigan resultados en forma temporal y cuyo desempeño es evaluado en tal sentido.

\section{Características centrales y contenido esencial de los programas de cumplimiento en la actualidad.}

\subsection{El carácter abierto y dinámico de los programas de cumplimiento.}

No se puede decir con precisión cuál es el contenido exacto de un programa de cumplimiento, lo que puede ser visto como una ventaja en la medida que su carácter abierto permitiría su continua adaptación a la realidad cambiante de la actividad empresarial, y también a la consideración de las diferenciaciones que se dan al interior de esta misma (debido a los diversos ámbitos en que se desarrolla). ${ }^{12}$ Con todo, esta amplitud y poca

\footnotetext{
${ }^{12}$ En este sentido GORDON, S., "Implementation of Effective Compliance and Ethics Programs and the Federal Sentencing Guidelines", en: VV. AA., Corporate Compliance Answer Book 2010, Estados Unidos: Ed. Practising Law Institute, 2010. Capítulo 2 disponible en Web:
} 


\section{Polít. crim. Vol. 8, № 16 (Diciembre 2013), Art. 6, pp. 544 - 573. [http://www.politicacriminal.cl/Vol_08/n_16/Vol8N16A6.pdf]}

claridad en su contenido también implicaría una mayor inseguridad jurídica en relación con la concreción de los deberes de la empresa que deben ir plasmados en estos mismos programas.

El contenido en concreto de estos instrumentos será variable de acuerdo al sector de la actividad empresarial del que se trate. Si bien se puede apreciar ciertas exigencias mínimas, es decir, aspectos a los cuales se debe atender a la hora de diseñarlos, pueden existir importantes diferencias, las que vendrían dadas no solo por las diversas actividades que ejecuten las empresas, sino también por las características estructurales de las mismas y la legislación en la que se desenvuelvan.

El último punto es de gran relevancia en la medida que estos programas deben tener por propósito el cumplimiento de la legislación aplicable en el marco de su actividad y, por ende, se encuentran destinados a prevenir defectos específicos derivados de ésta. Lo cual permite proponer una serie de pasos tendentes a la concreción de este contenido específico del sistema de prevención que implemente la empresa.

\subsection{Análisis del contenido del programa de cumplimiento atendiendo a ejemplos de la práctica internacional.}

Con el objeto de facilitar la exposición relativa al contenido de la práctica actual en materia de gestión de riesgos empresariales observaremos, solo a modo de ejemplo (ya que es una referencia especialmente útil), la estructura de un "modelo de organización, gestión y control" de una empresa con funcionamiento en Italia, que ha implementado tal sistema con el objeto de prevenir la sanción a la empresa por los delitos contenidos en el D. Lgs. 231/2001, que permite la imposición de responsabilidad administrativa en forma directa a la persona jurídica por una amplia gama de delitos en los casos en que éste sea cometido en interés de la misma o en su provecho por determinados sujetos que cumplen con los requisitos establecidos en la ley, relacionados con su posición jerárquica dentro de la organización. ${ }^{13}$

http://www.pli.edu/product_files/booksamples/26962_sample2.pdf, p. 34. Así, por ejemplo, Las Federal Sentencing Guidelines for Organizations (FSGO) solo señalan ciertas características que estos programas deben tener para que puedan ser considerados efectivos (única forma en que cumplen con su función atenuatoria de la responsabilidad de la empresa). Por ejemplo, la implicación de los altos directivos de la empresa en su implementación; el establecimiento adecuado de sistemas de selección y capacitación del personal, tanto en lo relacionado con la actividad desarrollada como en relación con los contenidos del programa de cumplimiento; la adopción de sistemas de auditoría interna; la existencia de sanciones disciplinarias al interior de la empresa, etc.. NIETO, La responsabilidad penal de las personas jurídicas, cit. nota ${ }^{\circ}$ 3, p. 192; LAUFER, W., "Corporate Liability, Risk Shifting, and the Paradox of Compliance", Vanderbilt Law Review, Vol. 52 (1999), pp. 1390 y ss. Al respecto, señala GÓMEZ-JARA DÍEZ, C., La responsabilidad penal de las Empresas en los EEUU, Sevilla: Ed. Fundación Ramón Areces, 2006, p. 77. Por ejemplo, que el contenido mínimo de estos instrumentos son: “(1) existencia de un código de conducta escrito; (2) supervisión de los esfuerzos de cumplimiento por parte del personal altamente cualificado; (3) no delegación de poderes discrecionales de las autoridades administrativas en personal con posible tendencia delictiva; (4) comunicación efectiva de los estándares y procedimientos contenidos en los códigos de conducta; (5) reforzamiento mediante procedimientos disciplinarios; (6) adopción de medidas adecuadas tras la detección de la infracción".

${ }^{13}$ En relación a los criterios para la imposición de responsabilidad contenidos en el D. Lgs. 231 italiano ver, 
ARTAZA, Osvaldo. "Sistemas de prevención de delitos o programas de cumplimiento. Breve descripción de las reglas técnicas de gestión del riesgo empresarial y su utilidad en sede jurídico penal"

La estructura de éste (que además representa una tendencia en los modelos italianos observados), se caracteriza por estar dividida en una introducción, una parte general y una parte especial, tal como se grafica en la siguiente tabla: ${ }^{14}$

\begin{tabular}{|c|c|}
\hline Unidad & Contenido \\
\hline Introducción & $\begin{array}{l}\text { Premisa } \\
\text { - Descripción de principios generales } \\
\text { contenidos en disposiciones legales } \\
\text { relacionadas a la imposición de responsabilidad } \\
\text { a la empresa. } \\
\text { - Exigencias legales para la implementación del } \\
\text { programa de cumplimiento. }\end{array}$ \\
\hline Parte General & $\begin{array}{l}\text { - Descripción de estructura del programa de } \\
\text { cumplimiento } \\
\text { - Principios generales de control interno } \\
\text { - Organismos de Vigilancia } \\
\text { - Difusión del programa de cumplimiento } \\
\text { - Reglas relativas a la modificación y } \\
\text { actualización del programa } \\
\text { - Sistema sancionatorio o disciplinario }\end{array}$ \\
\hline Parte Especial & $\begin{array}{l}\text { - Definición de las actividades riesgosas } \\
\text { (procesos sensibles) de la empresa } \\
\text { - Código Ético (Código de Conducta) } \\
\text { - Descripción de los procedimientos y } \\
\text { protocolos propios del programa } \\
\text { - Reglas específicas para la prevención de los } \\
\text { delitos en particular } \\
\text { - Descripción del tipo delictivo } \\
\text { - Principios generales de comportamiento } \\
\text { - Actividades riesgosas en particular y } \\
\text { - } \text { prescripciones específicas } \\
\text { - Sistema de delegación de poder }\end{array}$ \\
\hline
\end{tabular}

Si se observa la estructura descrita se puede distinguir fácilmente dos ámbitos bien diferenciados en el diseño de un modelo de gestión, organización y control. ${ }^{15}$ Estos ámbitos

entre otros, AMARELLI G., "I Criteri oggettivi di ascrizione del reato all'ente colectivo ed i reati in materia di sicurezza sul lavoro. Dalla teorica incompatibilità alla forzata convivenza", Diritto Penale Contemporaneo, pp. 1-10, http://www.penalecontemporaneo.it/upload/1366281036AMARELLI\%202013a.pdf, (última visita 20/05/2013). También, con más referencias, DE SIMONE, "La responsabilità da reato degli enti", cit. nota ${ }^{\circ}$ 2 , pp. 22 y ss.

${ }^{14}$ La estructura presentada a continuación corresponde al modelo de la empresa Fullsix Italia. Existe versión web del documento en http://www.fullsix.it/wpcontent/uploads/2013/09/v04_modello_organizzativo_parte_generale_draft_01.pdf

Tal estructura no quiere ser presentada en caso alguno como un ideal, solo se ha tomado como ejemplo con el objeto de estudiar los diversos aspectos que componen las reglas de gestión del riesgo penal en la actualidad.

${ }^{15}$ En la doctrina española, NIETO, La responsabilidad penal de las personas jurídicas, cit. nota $\mathrm{n}^{\circ} 3$, pp. 240 y ss., describe el contenido que debiera tener un modelo de organización, o Programa de Cumplimiento, distinguiendo por una parte la instauración de un "código de conducta" difundido adecuadamente y por la otra, el diseño e implementación de lo que se conoce como "sistema de control". 
reflejan a la perfección el contenido mínimo que debiera abarcar un programa de cumplimiento, o sistema de prevención de delitos. Es decir, satisface los niveles de preocupación que deben guiar el diseño de cualquier modelo de gestión de riesgos penales.

Estos son, por un lado, aquellos aspectos vinculados al reforzamiento de una cultura organizacional tendente al cumplimiento de la ley (es decir, al establecimiento de una serie de principios rectores afines al cumplimiento de la ley) y, por el otro, el de la instauración de los procedimientos y mecanismos en concreto para el cumplimiento de este propósito.

Que un programa de cumplimiento presente este contenido mínimo tiene que ver en gran parte con las prácticas asentadas en relación al control interno de la empresa y a la gestión de los riesgos propios de la misma, específicamente aquellos vinculados a lo que se conoce como "compliance". Así, por ejemplo, un contenido similar se puede observar en la metodología utilizada por un organismo especializado en control interno como es el Committee of Sponsoring Organizations of the Treadway Commission (COSO), ${ }^{16}$ que al momento de definir los aspectos que deben ser tenidos en cuenta a la hora de administrar los peligros de infracción al ordenamiento jurídico distinguen tanto una preocupación por el ambiente organizacional como por los aspectos netamente procedimentales. Especialmente relevantes para el objeto de estudio de este artículo son los estándares que propone esta institución bajo la denominación de Enterprise Risk Management.

Lo mismo se puede decir en relación a las U.S. Federal Sentencing Guidelines for Organizations (en adelante FSGO), ${ }^{17}$ de acuerdo con las cuales, para que la empresa se vea beneficiada con la reducción de la multa correspondiente luego de la comisión de un hecho delictivo debe haber implementado un programa efectivo para prevenir y detectar infracciones de ley. Esto supone satisfacer exigencias relacionadas con el diseño de estándares de cumplimiento y procedimientos que deben ser seguidos por los integrantes de la empresa y que justamente suponen intervenir tanto la estructura organizacional como el generar una cultura organizacional respetuosa con la legislación vigente.

\footnotetext{
${ }^{16}$ Para una descripción acabada de tales instrumentos, ver MOELLER, R., Coso Enterprise Risk. Establishing Effective Governance, Risk, and Compliance Processes, Ed. Wiley Corporate F\&A, 2011 (2 edición), Kindle Edition, Pos. 420 y ss.; LOUGHMAN, B.; SIBERY, R., Bribery and Corruption. Navigating the Global Risks, Ed. Wiley Corporate F\&A, Kindle Edition, 2012, Pos. 1323 y ss.

${ }^{17}$ Las FSGO contienen una serie de circunstancias que agravan y que aminoran en su caso la responsabilidad de la empresa para los casos en que se den los requisitos que contiene el mismo instrumento. Al respecto ver GÓMEZ-JARA, La responsabilidad penal, cit. nota $\mathrm{n}^{\circ} 12$, pp. 77 y ss. Escapa al ámbito de este artículo un estudio detallado de estas directrices, así como el análisis de aquellos factores que son tenidos en cuenta para agravar el monto de la multa a la empresa condenada. Más bien interesará acá únicamente una de las circunstancias que aminoran la responsabilidad penal de las empresas, esto es, que la misma disponga de un "programa ético y de cumplimiento efectivo". En relación con los efectos prácticos de contar con un Programa de Cumplimiento, NIETO, La responsabilidad penal de las personas jurídicas, cit. nota $\mathrm{n}^{\circ} 3$, $\mathrm{p}$. 191; HUFF, K., "The role of corporate compliance programs in determining corporate criminal liability: A suggested approach", Columbia Law Review, Vol. 96 (1996), pp. 1267 y ss.
} 
ARTAZA, Osvaldo. "Sistemas de prevención de delitos o programas de cumplimiento. Breve descripción de las reglas técnicas de gestión del riesgo empresarial y su utilidad en sede jurídico penal"

3.2.1. Primer nivel: el control del ambiente interno. importancia de fomentar una cultura organizacional tendente al cumplimiento de la ley.

Es usual que la primera preocupación detectada en un programa de cumplimiento sea la de reflejar que la observación del ordenamiento jurídico es una política corporativa y, por lo mismo, se presenta a los integrantes de la empresa como una de las preocupaciones prioritarias de la alta dirección de la empresa, junto a la obtención de beneficios y el logro de los objetivos de la organización.

Así, por ejemplo, el modelo de organización, gestión y control observado empieza con una "premessa" dentro de la cual se manifiesta que uno de los objetos del "modelo" es precisamente potenciar un comportamiento correcto y transparente en sus destinatarios con el objeto de prevenir las conductas delictivas contenidas en el D. Lgs. 231 de 2001.

Para lo mismo, se observan una serie de medidas tendentes a asegurar el fomento de una cultura organizacional respetuosa con la ley, destacándose por su relevancia práctica la presencia de un código de ética o de conducta, por medio del cual se explicita el conjunto de valores propios de la organización, así como un sistema de capacitación e interiorización del modelo conducente a reforzar tal forma de comportamiento. ${ }^{18}$ En este sentido, se ha señalado en España, por ejemplo, que el fin primordial de este instrumento debe ser "promover una cultura dentro de la organización que más allá de asegurar el cumplimiento de la ley, anime a que la actividad en general de la empresa se corresponda con determinados patrones éticos". 19

\footnotetext{
${ }^{18}$ Para NIETO, La responsabilidad penal de las personas jurídicas, cit. nota $\mathrm{n}^{\circ}$ 3, pp. 240 y ss., Se podría identificar al Código de Conducta, "con un conjunto de disposiciones que tienen como finalidad reforzar los mandatos que se contienen en un texto legal. Es lo que se conoce con el nombre de compliance. Éstos "constituyen el tronco del sistema de organización interno, sobre él descansan el resto de contenidos".

${ }^{19}$ NIETO, La responsabilidad penal de las personas jurídicas, cit. nota $\mathrm{n}^{\circ} 3$, p. 241 . En general se puede decir que sus principales funciones son: a) Refuerzo del mandato normativo y la asignación de relevancia al cumplimiento de la ley como uno de los objetivos prioritarios de la organización. b) Cumplir con una función de especificación o concreción de las normas jurídicas, "mostrando a sus destinatarios la forma en que debe actuar el empleado ante una oportunidad delictiva o una situación de riesgo para determinados bienes jurídicos". Esto implica que deben estar redactados en términos los suficientemente flexibles como para ir adaptándose a nuevas situaciones que se vayan presentando en el ejercicio propio de la actividad de la empresa. Se suele decir, además, que este tipo de instrumentos sirven para "reducir la complejidad" vinculada al comportamiento de acuerdo con la norma penal en determinados ámbitos de regulación relativamente complejos. Esta concreción del contenido de la norma viene dado por la definición del cómo cumplir con la exigencia legal. c) Cumplirían con una función externa (destinada a sujetos fuera de la estructura organizacional) vinculada a la regulación de la relación de la empresa con otros organismos y al respeto de su normativa interna en esta interacción. Ejemplos claros en donde esta finalidad es del todo relevante serían los de la lucha contra la corrupción, en donde se pretende evitar que las empresas "cierren los ojos" ante determinadas ilegalidades que se puedan cometer en la contratación con empresas ubicadas en el extranjero mediante la delegación de la tarea de contratar con éstas, o también el sector productivo en relación a la contratación con proveedores con el objeto de impedir, por ejemplo, que el suministro de determinados bienes se haya obtenido mediante trabajo infantil, etc. d) En éste se fijarían las normas de comportamiento de la empresa en forma general, por ende, contendría no solo el reforzamiento del mandato ya sea penal o proveniente de otra área del ordenamiento jurídico, sino que puede ir más lejos en el sentido de establecer, por ejemplo, los procedimientos tendentes a regular la conducta esperada luego de la comisión de un ilícito o los procedimientos necesarios para poder advertir de la comisión de una conducta delictiva, etc. Ver al respecto
} 


\section{Polít. crim. Vol. 8, No 16 (Diciembre 2013), Art. 6, pp. 544 - 573. \\ [http://www.politicacriminal.cl/Vol_08/n_16/Vol8N16A6.pdf]}

Si se observa un código de ética es común notar que exprese, en primer lugar, una serie de objetivos corporativos y la forma en que los integrantes de la empresa pueden alcanzar los mismos. Esta actividad debe enmarcarse o regirse por este conjunto de valores y principios que determinan el marco de acción de los integrantes de la organización.

Esta tendencia responde justamente a una serie de prácticas asentadas en el sector del control interno de las organizaciones empresariales, como se podrá observar, que considera que para los efectos de prevenir conductas delictivas es imprescindible incentivar un ambiente al interior de la empresa que fomente el comportamiento ajustado al ordenamiento jurídico como manifestación de ciertos valores compartidos y debidamente interiorizados por los integrantes de la empresa.

Como se expresó más arriba, es usual sostener que el cumplimiento de la ley debe ser entendido por la dirección de la empresa como uno más de los problemas de gestión que se le presentan en forma cotidiana en el manejo de sus operaciones y en la búsqueda del cumplimiento de sus objetivos. Por lo mismo, COSO, dentro de su "estructura de control interno" considera, especialmente, el nivel "compliance" como una de sus categorías más relevantes. Incluso, dentro de su propuesta metodológica o modelo para el control interno, incluye como uno de sus principales niveles el del "control del entorno" (control environment foundation level), como base de todo control interno que pueda presentar la empresa, ya que será este nivel el que influirá en como se estructuran los negocios de la empresa y como se evalúen sus propios riesgos. ${ }^{20}$

De acuerdo a tal punto de partida, todo componente del control interno de la organización empresarial se funda en este nivel, ya que refleja la "actitud general", la "conciencia" de la misma y, especialmente, la disposición y las actividades dirigidas hacia el control interno derivadas desde su alta dirección (lo que se conoce como tone at the top). Por lo mismo, no se puede pretender una gestión del riesgo eficiente sin influir a su vez en este nivel, generando una cultura organizacional que tienda a la observancia del ordenamiento jurídico.

Uno de los aportes más relevantes que se derivan de estas recomendaciones guarda relación con la identificación de factores que inciden en el control del entorno organizacional. El primero de estos, y el más relevante desde la perspectiva analizada, es el de la "integridad y los valores éticos" de la empresa. En la actualidad esta tendencia se ve reflejada con la presencia de los códigos de ética de la organización que reflejarían tales valores compartidos por la misma. $^{21}$

también, GORDON, “Implementation”, cit. nota n 12, pp. 43 y ss. Ver también recientemente en España, BACIGALUPO ZAPATER, E., Compliance y Derecho penal, España: Ed. Aranzadi, 2011, pp. 45-60. Otros ámbitos en que esta finalidad de los Códigos de conducta es de gran relevancia serían el de prevención de riesgos laborales (art. 2 del RD 39/1997), el de Sanidad Alimentaria, por medio del Sistema de Análisis de Peligros y Puntos Críticos (RD 2207/1995), o en materia Medio ambiental, por medio de los sistemas de gestión exigidos por el R CEE 1836/93.

${ }^{20}$ Ver al respecto, MOELLER, Coso Enterprise, cit. nota n ${ }^{\circ} 16$, Pos. 458.

${ }^{21}$ Se debe incluir dentro de este componente de acuerdo a MOELLER, Coso Enterprise, cit. nota ${ }^{\circ} 16$, pos. 1830, entre otros, los siguientes elementos: a) Filosofía de la gestión del riesgo que subyace a las prácticas cotidianas de la empresa, b) El "apetito de riesgo", que representa la cantidad de riesgo que se está dispuesto a 
ARTAZA, Osvaldo. "Sistemas de prevención de delitos o programas de cumplimiento. Breve descripción de las reglas técnicas de gestión del riesgo empresarial y su utilidad en sede jurídico penal"

Con todo, no es el objeto de este artículo analizar ni describir el conjunto de recomendaciones que provienen de esta organización, sino que solo el de evidenciar un conjunto de principios mínimos para la gestión del riesgo que debieran ser considerados a la hora de definir y delimitar las expectativas que se dirigen a las empresas en la actualidad relativas a los controles que deben implementar para reducir sus riesgos penales. Como se pudo observar, uno de los niveles que no pueden ser descuidados es el del entorno o el del ambiente organizacional, específicamente en lo que se refiere al refuerzo de una cultura respetuosa del orden jurídico.

Desde la perspectiva de la prevención de riesgos penales derivados de la actividad de la empresa, destacan por su importancia las FSGO. El informe del "Advisory Group" sostiene que uno de los principales propósitos de éstas sería el de crear incentivos (mediante el establecimiento de consecuencias vinculadas al comportamiento meritorio o reprochable de la organización) para el refuerzo del cumplimiento de la ley por parte de la empresa. ${ }^{22}$ Este objetivo se vería facilitado o aumentarían sus posibilidades de satisfacción, según las conclusiones del mismo grupo, cuando está integrado dentro de la cultura organizacional.

Se ha sostenido que una cultura organizacional es el conjunto de reglas y principios que guían el comportamiento individual y grupal que han sido determinados por la dirección de la empresa y que son expresados como "valores compartidos por la organización" o principios rectores, reforzados por una serie de procedimientos desarrollados en ésta. En suma, que ésta "invita al cumplimiento de la ley" cuando este tipo de comportamiento es considerado como una "conducta esperada". ${ }^{23}$

De hecho, este sería el propósito de una serie de reformas introducidas en Estados Unidos, en la primera década de este siglo (por ejemplo, la Sarbanes-Oxley Act de 2002) principalmente con el objeto de reforzar la adopción de códigos de conducta que incluyan un estándar en el comportamiento ético mínimo esperado por la organización. ${ }^{24} \mathrm{Al}$ parecer,

aceptar en la organización, c) Las actitudes de la Dirección relativa al riesgo, d) Integridad y valores compartidos, e) etc.

${ }^{22}$ En este sentido, el Report of the ad hoc advisory group on the organizational sentencing guidelines del 710-03, p. 51, http://www.ussc.gov/Guidelines/Organizational_Guidelines/advgrprpt/advgrprpt.htm, (última visita 20/05/2013).

GÓMEZ-JARA, La responsabilidad penal, cit. nota $\mathrm{n}^{\circ}$ 12, p. 125; GORDON, "Implementation", cit. nota $\mathrm{n}^{\circ}$ 12, p. 41; SHARP PAINE, L., "Managing for Organizational Integrity", Harvard Business Review, MarzoAbril 1994, pp. 106 y ss.

${ }^{23}$ Report of the ad hoc advisory group, cit. nota $\mathrm{n}^{\circ} 22$, p. 52. En España se refiere a este aspecto de la actividad empresarial, entre otros, BACIGALUPO, Compliance y Derecho penal, cit. nota ${ }^{\circ}$ 19, pp. 17-19.

${ }^{24}$ En relación con la génesis, objetivos y críticas a este instrumento ver HEFENDEHL, R., "Enron, WorldCom, and the Consequences: Business Criminal Law Between Doctrinal Requirements and the Hopes of Crime Policy", Buf. Crim. L. Rev., Vol. 8 (2005), pp. 51-54; SALVADOR CODERCH, P.; GÓMEZ LIGÜERRE, C., "Respondeat Superior II. De la responsabilidad por hecho de otro a la responsabilidad de la organización", InDret, Julio 2002, pp. 1 y 22, http://www.indret.com/pdf/088 es.pdf (última visita 20/05/2013).; FOFFANI, L., "Escándalos económicos y reformas penales: Prevención y represión de las infracciones societarias en la era de la globalización", $R P \mathrm{n}^{\circ} 23$ (2009), pp. 35 y ss.; GORDON, "Implementation", cit. nota $\mathrm{n}^{\circ}$ 12, pp. 35 y ss.; SCHÜNEMANN, B., "The Sarbanes-Oxley Act of 2002: A German Perspective”, Buffalo Criminal Law Review, Vol. 8 (2004), pp. 43 y ss., con un énfasis diverso en la descripción de este instrumento destacando la relevancia que se le asignaría a la responsabilidad individual. 


\section{Polít. crim. Vol. 8, № 16 (Diciembre 2013), Art. 6, pp. 544 - 573. \\ [http://www.politicacriminal.cl/Vol_08/n_16/Vol8N16A6.pdf]}

la adopción de un código de conducta, en el que se refleja esta cultura empresarial, se ha transformado en uno de los pilares de un programa de cumplimiento efectivo.

Solo resta señalar al respecto que la tendencia actual es que no basta con la existencia de un código de conducta si éste no se haya lo suficientemente interiorizado en los trabajadores de la organización, y especialmente en sus altos cargos. ${ }^{25}$

Sin embargo, como es evidente, no basta con la descripción de una serie de conductas esperadas o valores compartidos si no se establece de forma adecuada el modo en que éstas se llevarán a cabo, es decir, como se asegurará su cumplimiento. ${ }^{26}$

\subsubsection{Segundo nivel: las reglas procedimentales de gestión o administración del riesgo.}

Uno de los aspectos centrales del programa de cumplimiento que ha servido como ejemplo, al menos como se puede desprender de la simple vista de su estructura, sería la adopción de aquellos mecanismos necesarios para asegurar que dentro de la empresa se cumpla con el mandato legal. Por lo mismo en su Parte general describe sus principios de control interno, establece sus organismos de vigilancia, su sistema sancionatorio, y en su Parte especial instaura una serie de protocolos y procedimientos internos para evitar que sus integrantes cometan delitos.

Es importante reconocer que no se puede establecer de forma categórica un estilo único de procedimientos o mecanismos que puedan operar para todo tipo de empresas, ya que la eficacia de estos dependerá, y esta es la tendencia actual que se ve corroborada por las modificaciones que se han desarrollado en las FSGO, de las características internas de la empresa (como su tamaño y su complejidad) y también por la función económica que cumple y el sector en el que se desarrolla. ${ }^{27}$

Por lo mismo, las FSGO no imponen un sistema de control en específico, tarea que corresponde a la propia organización, sino que, más bien, señala una serie de pasos o aspectos que deben ser considerados a la hora de evaluar ese sistema como adecuado o no. Es decir, establece los criterios que son esperables de cualquier sistema de control adoptado por la empresa y que deben ser revisados por el juez.

\footnotetext{
${ }^{25}$ Al respecto ver entrevista a Michael Toffel, en 2007 para Harvard Business School; "Industry SelfRegulation: What's Working (and What's Not)?", en donde se refuerza la idea de que la sola adopción de un Código de conducta no es suficiente sin otros mecanismos complementarios. Disponible en Web: http://hbswk.hbs.edu/item/5590.html (última visita 26/08/2013).

${ }^{26}$ Ver al respecto GORDON, "Implementation", cit. nota $\mathrm{n}^{\circ} 12$, p. 50, con referencias directas al caso "Enron" y la falta de sustancia que presentaría el Código de conducta de la misma, la que solo sería un "mero papel". Además HECHLER BAER, M., "Governing Corporate Compliance", Boston College Law Review, Vol. 50 (2009), pp. 4 y ss., especialmente en relación con la evolución en el ámbito angloamericano para evitar el problema del "cosmetic compliance" (o programas de cumplimiento de "mero papel" como se les suele denominar).

${ }^{27}$ En relación con la relevancia del "tamaño" y complejidad de la empresa, GORDON, "Implementation", cit. nota $\mathrm{n}^{\circ} 12$, pp. 34-35.
} 
ARTAZA, Osvaldo. "Sistemas de prevención de delitos o programas de cumplimiento. Breve descripción de las reglas técnicas de gestión del riesgo empresarial y su utilidad en sede jurídico penal"

Con todo, si bien no conviene otorgar un contenido específico a tal sistema, sí se pueden derivar de la práctica una serie de criterios mínimos que debe contener cualquier sistema de prevención de delitos o modelo de gestión que quiera responder a un nivel mínimo de eficiencia. Éstos, al igual como se hizo con anterioridad, pueden ser detectados de la tendencia actual en esta materia, a partir de la cual es posible extraer una serie de elementos fundamentales para efectos de administrar adecuadamente el riesgo.

Una de las principales características del modelo de gestión que se ha elegido como ejemplo, es que la empresa no define las medidas que adoptará para prevenir sus delitos sin antes haber evaluado seriamente cuales son las actividades riesgosas que se generan a propósito de su misma actividad. Por lo mismo, dentro de sus principios fundamentales de control interno incluyen tanto el proceso de "Risk Assessment" como se denomina en la práctica angloamericana así como el de "Risk Mitigation".

Un modelo efectivo de prevención de delito debe responder, entonces, a aquellas actividades de riesgo que son propias de la actividad empresarial de la que se trate, lo que refuerza aún más la idea de que el deber de prevenir conductas delictivas que recae en la empresa no es absoluto, sino que reducido a cierto ámbito de competencia dado por aquello que provenga del giro de la organización.

En este ámbito es especialmente relevante y útil observar la metodología propuesta por COSO para la evaluación de los riesgos potenciales propios de la actividad de la que se trate. De acuerdo a esta metodología, el proceso de gestión de riesgos (en un sentido amplio y, por ende, no solo aquellos asociados al área de Compliance) debe incluir la definición de aquellos que deben ser controlados. A esta actividad se la conoce como "planificación del riesgo" y consiste principalmente en proyectar toda la gama de riesgos a los que se expone la actividad de la empresa. Evidentemente, para lo que nos ocupa, solo serán relevantes los asociados al incumplimiento de la ley, y especialmente de la ley penal.

Una vez que la empresa a "planificado" el riesgo, comienzan una serie de fases o etapas que componen lo que se denomina como "Risk Management". Estas son: a) Identificación del riesgo; b) Evaluación cualitativa y cuantitativa del riesgo; c) Priorización del riesgo y planificación de la respuesta al mismo; e) Monitoreo del riesgo.

La primera de éstas consiste en la "identificación del riesgo", mediante la cual la empresa debiera definir las diferentes actividades riesgosas que se efectúan a lo largo de su organigrama, identificando, a su vez, los integrantes de la empresa que se exponen a las mismas.

Con todo, y de acuerdo a la metodología estudiada, no basta con la identificación de riesgos, ya que si el fin de tal fase es la de adoptar las medidas tendentes para controlarlos, se debe considerar una segunda fase de evaluación de éstos (en forma cualitativa y cuantitativa), que en síntesis consiste en definir la magnitud del mismo, lo que se efectúa por lo regular, atendiendo tanto a la probabilidad que presenta el riesgo en particular como su impacto potencial. Por obvio que parezca, no tiene mucho sentido reaccionar igual frente 


\section{Polít. crim. Vol. 8, № 16 (Diciembre 2013), Art. 6, pp. 544 - 573. [http://www.politicacriminal.cl/Vol_08/n_16/Vol8N16A6.pdf]}

a cualquier riesgo sin considerar en primer lugar la gravedad del mismo y la probabilidad de que ocurra. Un riesgo de mínima probabilidad y de un impacto muy bajo no debe ser controlado como uno de mayor probabilidad y de fuerte impacto. ${ }^{28}$

Siguiendo con las referencias a los instrumentos elegidos para describir la tendencia actual en esta materia, conviene considerar lo que el "Advisory Group" ha señalado a propósito del análisis de las FSGO. Según estas recomendaciones, un factor fundamental para evaluar la efectividad de un programa de cumplimiento es si presenta o no estándares de conducta y un sistema de control interno que se manifieste como capaz de reducir las infracciones a la ley que sean más frecuentes o típicas del sector en concreto. Esta perspectiva tiene la ventaja de dar la suficiente relevancia a estos procedimientos y, además, enfatiza que la medida de eficacia debe venir dada en términos de su impacto en la reducción de ilícitos propios (típicos o más frecuentes, de acuerdo a la experiencia) del sector de la empresa. ${ }^{29} 30$

Por ende, un programa de cumplimiento que no guarda relación con la gestión de aquellos riesgos más relevantes vinculados a los peligros ya identificados de su actividad, nunca podría ser catalogado como efectivo ya que falla en su núcleo. Así mismo, la evaluación de riesgos implica u obliga una constante actualización, ya que el modelo dejaría de cumplir su fin primordial, al no responder a la práctica actual de la empresa.

Con todo, la evaluación de riesgos es solo un paso inicial (aunque se vaya acudiendo constantemente a éste en una especie de ciclo), ya que luego de determinar los riegos propios de la actividad se inicia una tercera fase de planificación de la respuesta al riesgo, mediante la cual se deben generar los procedimientos y mecanismos adecuados para su control.

Si se observa la estructura de programa de cumplimiento descrita más arriba, se denota en su parte especial la intención de dotar a los integrantes de la empresa de protocolos específicos de comportamientos para hacer frente a actividades riesgosas detectadas en la etapa de levantamiento de riesgos en forma de respuestas desde la empresa para cada uno de los delitos por los cuales puede responder la empresa. Es decir, cómo debe actuar el integrante de la misma al verse expuesto a los riesgos detectados.

Por lo mismo, COSO recomienda que una vez se hayan evaluado los riesgos se defina la clase de respuesta a la que se optará en relación a éste. Dentro de la gama de respuestas posibles solo interesan acá las que tengan que ver con la reducción o control del riesgo. ${ }^{31}$ Para tales efectos es fundamental el concepto de "actividades de control", entendido como el conjunto de políticas y procedimientos necesarios para asegurar que las respuestas para los riesgos identificados se apliquen en forma efectiva al interior de la empresa.

\footnotetext{
${ }^{28}$ MOELLER, Coso Enterprise, cit. nota ${ }^{\circ} 16$, Pos. 1361.

${ }^{29}$ Report of the ad hoc advisory group, cit. nota $\mathrm{n}^{\circ} 22$, p. 56 y p. 87, según los cuales, las evaluaciones de riesgo deben realizarse en todas las etapas del desarrollo, prueba e implementación de un programa de cumplimiento para asegurar que el esfuerzo de cumplimiento sea efectivo y esté bien coordinado.

${ }^{30}$ Con ejemplos en el área de la salud, Report of the ad hoc advisory group, cit. nota n ${ }^{\circ} 22$, p. 89.

${ }^{31}$ MOELLER, Coso Enterprise, cit. nota ${ }^{\circ} 16$, Pos. 2220.
} 
ARTAZA, Osvaldo. "Sistemas de prevención de delitos o programas de cumplimiento. Breve descripción de las reglas técnicas de gestión del riesgo empresarial y su utilidad en sede jurídico penal"

El modelo que se utiliza de ejemplo refleja correctamente esta exigencia al incluir dentro de su estructura interna (de control, gestión y control) una serie de procedimientos dirigidos a la prevención de los delitos en particular contenidos en la legislación italiana y por los cuales puede responder administrativamente la empresa. Dentro de estos destacan por su relevancia: a) protocolos de formación del personal, b) gestión de reclamaciones y denuncias internas, c) participación en licitaciones y contratación con la Administración Pública, d) operaciones económicas con terceros, e) operaciones contables, presupuesto y balance, descripción del sistema de información, f) manual de procedimientos de sistemas de calidad, g) reglamento interno, etc. ${ }^{32}$

Al respecto resulta muy útil atender a ciertas características de las actividades de control que se extraen desde las FSGO. De tal instrumento se pueden rescatar fácilmente ciertos principios organizativos que deben ser considerados a la hora de diseñar un programa de cumplimiento. A continuación nos remitiremos exclusivamente a estas directrices.

a) Involucramiento de la alta dirección de la empresa en labores de supervisión y control y delegación adecuada de funciones de vigilancia.

Una de las primeras preocupaciones que se obtiene de este instrumento guarda relación con el papel que juegan los administradores de la empresa y sus altos cargos. ${ }^{33}$ Se pretende que este sector de la empresa se involucre de manera activa con la supervisión, al menos, del sistema de control que adopte la empresa. ${ }^{34}$ Lo anterior se contiene de la siguiente forma en las FSGO (Sección 8B2.1.):

“b. 2. A. La directiva de la organización deberá tener conocimiento del contenido y la ejecución del programa ético y de cumplimiento, y deberá ejercer una supervisión razonable con respecto a la implementación y efectividad del programa ético y de cumplimiento".

"B. El personal de alta dirección de la organización deberá asegurarse de que la organización tiene un programa ético y de cumplimiento efectivo, tal como se describe en esta Directriz. Deberá asignarse una responsabilidad genérica por el programa ético y de cumplimiento efectivo a determinado(s) individuo(s) dentro del personal de alta dirección".

"C. Deberá delegarse a determinado(s) individuo(s) dentro de la organización la responsabilidad por la ejecución diaria del programa ético y de cumplimiento efectivo. Los individuos que respondan de la ejecución deberán dar cuentas diariamente al personal de alta dirección y, en su caso, a la directiva o a un comité de la directiva, en torno a la efectividad del programa ético y de cumplimiento. Para desempeñar dicha

\footnotetext{
${ }^{32}$ Ver al respecto p. 50 Modelo de organización, gestión y control empresa Fullsix, nota ${ }^{\circ} 14$.

${ }^{33}$ En este sentido GORDON, "Implementation", cit. nota n ${ }^{\circ} 12$, pp. 32-33.

${ }^{34}$ Report of the ad hoc advisory group, cit. nota ${ }^{\circ} 22$, pp. 59-60. Así, por ejemplo, una de las modificaciones propuestas por el "Advisory Group" es la inclusión de una referencia directa al involucramiento de los altos ejecutivos en esta labor, lo que se desprende de su propuesta de redacción de la Sección 8B2.1(b)(2) de las FSGO.
} 


\section{Polít. crim. Vol. 8, № 16 (Diciembre 2013), Art. 6, pp. 544 - 573. [http://www.politicacriminal.cl/Vol_08/n_16/Vol8N16A6.pdf]}

responsabilidad se dotará al individuo de los recursos adecuados, la autoridad apropiada y el acceso directo a la directiva o al comité de la directiva". 35

Como se desprende de tales disposiciones, este principio implica, por una parte, un verdadero involucramiento de la alta dirección con el programa de cumplimiento, lo que se refleja en diversos frentes. El primero de éstos guarda relación con el efectivo conocimiento del sistema de organización, lo que se corresponde, además, con la necesidad de que sus principios y contenido mínimo se diseñen con el grado adecuado de empoderamiento desde este sector de la empresa. En segundo lugar, no solo basta que la alta dirección conozca, sino que debe mantener cierta cuota de responsabilidad en su diseño e implementación. Es responsabilidad de este sector que la empresa cuente con tal programa así como el de definir aquellos integrantes de la alta dirección encargados de velar por el cumplimiento general de éste. ${ }^{36}$ Por último, se exige que sea este sector el que delegue, con ciertas limitaciones, la supervisión del funcionamiento cotidiano del sistema en concreto. Este mandato se encuentra restringido en razón de la exigencia de comunicación diaria a la alta dirección, acerca del efectivo funcionamiento del modelo organizativo.

En consonancia con tal principio, la empresa que ha servido de ejemplo contempla dentro de sus definiciones estructurales aparejadas a la implementación del modelo la incorporación de un organismo de vigilancia caracterizado, al menos en su diseño, por su autonomía e independencia, su profesionalismo y por efectuar una labor constante (continuidad de su accionar). Dentro de lo que interesa ahora, señala el modelo que el requisito de la autonomía e independencia puede ser satisfecho mediante una colocación elevada de tal organismo dentro de la estructura jerárquica de la empresa, así como

\footnotetext{
${ }^{35}$ Traducción tomada de GÓMEZ-JARA, La responsabilidad penal, cit. nota ${ }^{\circ} 12$, pp. 125 y ss. Al respecto, ver también documentos preparados por el "Ethics Resource Center" en agosto de 2007, "Leading Corporate Integrity: Defining the Role of the Chief Ethics and Compliance Officer", en p. 2 describe la tendencia actual de que muchas organizaciones empresariales optarían por consolidar su sistema de prevención y detección de conductas delictivas, a través de un "jefe de ética y cumplimiento (CECO, o "chief ethics and compliance officer"). Con todo, las responsabilidades específicas de éste no se presentan, por lo general, en forma clara y en muchas ocasiones no estarían dotados ni de la autoridad ni de los recursos suficientes como para cumplir tal labor. Resulta útil, además, considerar ciertos requisitos mínimos de estos "ofíciales de cumplimiento". En primer lugar, se sostiene que para que esta función se considere con algún "valor añadido" debe presentar un equilibrio tanto entre la adaptación a las características únicas de la organización con aquellas herramientas mínimas necesarias para cumplir con su función (recursos, autoridad, etc.). Sostienen que como mínimo el "CECO" debe ser responsable ante la junta directiva de la empresa de las obligaciones que se han delegado en éste, lo que se debe hacer sin temor de represalias y advirtiendo los eventuales conflictos de interés que se pueden presentar. Debe guiar a las operaciones de la empresa en orden a la generación de una cultura ética o de cumplimiento y debe contar con la posibilidad de tomar decisiones de relevancia y de llevar a cabo recomendaciones que deben ser tomadas en serio en todos los niveles de la organización. Disponible en web: http://www.ethics.org/files/u5/RPceco.pdf (última visita 20/05/2013).

${ }^{36}$ En forma expresa el Art. $4^{\circ}$, Ley 20.393 chilena: "Modelo de prevención de los delitos. Para los efectos previstos en el inciso tercero del artículo anterior, las personas jurídicas podrán adoptar el modelo de prevención a que allí se hace referencia, el que deberá contener a lo menos los siguientes elementos: 1) Designación de un encargado de prevención. a) La máxima autoridad administrativa de la persona jurídica, sea ésta su directorio, un socio administrador, un gerente, un ejecutivo principal, un administrador, un liquidador, sus representantes, sus dueños o socios, según corresponda a la forma de administración de la respectiva entidad, en adelante la "Administración de la Persona Jurídica", deberá designar un encargado de prevención, quien durará en su cargo hasta tres años, el que podrá prorrogarse por períodos de igual duración".
} 
ARTAZA, Osvaldo. "Sistemas de prevención de delitos o programas de cumplimiento. Breve descripción de las reglas técnicas de gestión del riesgo empresarial y su utilidad en sede jurídico penal"

permitiendo que se comunique, para fines informativos, en forma directa e inmediata con el "Consiglio di Amministrazione".

b) Comunicación y formación continua de los integrantes de la empresa.

Las FSGO disponen en relación a este principio lo siguiente:

"4.A. La organización deberá adoptar medidas razonables para comunicar periódicamente, y de manera práctica, sus estándares y procedimientos, además de otros aspectos de su programa ético y de cumplimiento, a los individuos que están referidos en el subapartado (B), llevando a cabo programas efectivos de entrenamiento y distribuyendo de otras maneras la información que resulte apropiada para los roles y responsabilidades que corresponden a dichos individuos".

"B. Los individuos referidos en el subapartado (A) son los miembros de la directiva, el personal de la alta dirección, el personal con autoridad significativa, los empleados de la organización y, en su caso, los representantes de la organización".

Como se desprende de lo anterior, un aspecto que también debe ser considerado a la hora de diseñar un Programa de Cumplimiento se relaciona con el grado de conocimiento y entrenamiento con el que cuentan determinados integrantes de la empresa referido a los procedimientos existentes de carácter interno. ${ }^{37}$ Con especial énfasis en las medidas adoptadas para que quienes poseen cargos con mayor responsabilidad estén adecuadamente actualizados en este sentido. Una vez más, la forma en que la empresa cumpla con estos requerimientos (conocimiento efectivo y constante actualización o entrenamiento) será de su exclusiva responsabilidad. ${ }^{38}$

Con todo, actualmente se considera que la obligación de comunicación efectiva no se satisface con la mera publicación de los procedimientos existentes al interior de la empresa relacionados con el aseguramiento del cumplimiento de la ley. Lo anterior debe ser complementado con la apropiada motivación a sus integrantes vinculada al seguimiento de tales procedimientos ${ }^{39}$.

c) Adopción de mecanismos de monitoreo y evaluación del programa.

En tercer término, luego de la exigencia de involucramiento y de conocimiento efectivo, las FSGO establecen ciertos mecanismos vinculados con formas de asegurar el cumplimiento

\footnotetext{
${ }^{37}$ GORDON, "Implementation", cit. nota $\mathrm{n}^{\circ} 12$, p. 33.

${ }^{38}$ Report of the ad hoc advisory group, cit. nota $n^{\circ} 22$, p. 69 , señalan que la empresa debe tener la flexibilidad para determinar el tipo de programa de entrenamiento que quiera adoptar y cómo hará circular debidamente la información de acuerdo al tamaño de la organización y el tipo de conductas delictivas que podrían ser cometidas en el marco del desarrollo de su actividad.

${ }^{39}$ Report of the ad hoc advisory group, cit. nota $\mathrm{n}^{\circ} 22$, p. 70 y 71 . En términos muy diversos la Ley 20.393 (Chile), que al parecer exige únicamente una comunicación formal a los integrantes de la empresa por medio de la incorporación de las diversas obligaciones que derivan del sistema de prevención de delitos en los respectivos reglamentos y contratos de trabajo (art. 4. 3) d)).
} 


\section{Polít. crim. Vol. 8, N 16 (Diciembre 2013), Art. 6, pp. 544 - 573. \\ [http://www.politicacriminal.cl/Vol_08/n_16/Vol8N16A6.pdf]}

de los procedimientos que tiendan a evitar hechos ilícitos, mediante la incorporación de la exigencia de monitoreo, auditorías y evaluación.

"5. La organización deberá adoptar medidas razonables para:

A. Asegurarse de que se observa el programa ético y de cumplimiento, incluyendo controles y auditorías para detectar la conducta delictiva.

B. Evaluar periódicamente la efectividad del programa ético y de cumplimiento...".

Esta exigencia es una de las que ha adquirido mayor importancia en la práctica y la que ha tenido mayor desarrollo, sobre todo por la necesidad de que los programas de cumplimiento puedan adaptarse a las nuevas realidades que se presenten en el ejercicio de la actividad de la empresa y así poder revisar constantemente la idoneidad de los procedimientos diseñados por ésta y asegurar la pronta corrección de las falencias que se puedan detectar.

Por ejemplo, el "Advisory Group" da cuenta de este acuerdo en los expertos, relativo a lo esencial que resulta para el éxito del programa de cumplimiento, la incorporación de un sistema regular de evaluación en la medida que provee a la empresa de la flexibilidad necesaria para mantener ciertos procedimientos bases, aun cuando puedan adaptar otros mecanismos a circunstancias especiales que puedan darse en su interior. ${ }^{40}$

Se señala al respecto, que una evaluación y auditoría regular de los progresos y éxitos de un programa de cumplimiento es un paso muy importante en el aseguramiento de que éste se encuentra adecuadamente enfocado y conducido para su eficacia general como medio de detección y prevención de conductas delictivas.

A grandes rasgos, se pueden dar algunas características generales de esta exigencia en el marco de las FSGO. En primer lugar, se considera necesario la existencia tanto de un sistema de monitoreo interno que funcione de manera permanente como de un procedimiento de auditoría que responda a una perspectiva externa e independiente de la eficacia de tales procedimientos. Ambos métodos son necesarios para la consideración de un programa de cumplimiento como efectivo.

En segundo lugar, se ha señalado que la organización debe examinar separadamente en detalle y en forma regular dos características de la organización: ${ }^{41}$

i) La adhesión (o grado de internalización efectivo) a las leyes aplicables a la actividad de la empresa y a los requerimientos propios del modelo y;

\footnotetext{
${ }^{40}$ Report of the ad hoc advisory group, cit. nota $\mathrm{n}^{\circ} 22$, p. 75. Este grupo da algunos ejemplos de directrices vinculadas a este aspecto dadas en determinados sectores de gran relevancia, y que pueden servir para facilitar la comprensión de los fines que se pretenden con esta exigencia. Así, por ejemplo, aquellos estándares diseñados por el “International Standard Organization for an environmental management system” (EMS), cuyo propósito esta dado, en parte, para asegurar el cumplimiento de la regulación medioambiental y que requiere que tal sistema incluya medidas de monitoreo, auditoría y de evaluación. Otro ejemplo útil es el dado en el marco de la "Health Care Association" que ha propuesto algunos criterios para evaluar la calidad de los programas de cumplimiento en organizaciones hospitalarias o clínicas, pp. 74 y ss.

${ }^{41}$ Report of the ad hoc advisory group, cit. nota $\mathrm{n}^{\circ} 22, \mathrm{p} .76$.
} 
ARTAZA, Osvaldo. "Sistemas de prevención de delitos o programas de cumplimiento. Breve descripción de las reglas técnicas de gestión del riesgo empresarial y su utilidad en sede jurídico penal"

ii) La suficiencia o idoneidad de las prácticas comprendidas en el programa y que correspondan a la administración de la organización vinculadas a asegurar una razonable medida de éxito en la prevención y detección de conductas delictivas.

Desde el análisis de las FSGO, llevado a cabo por la doctrina y jurisprudencia norteamericana, se ha señalado que el monitoreo, la práctica de auditorías y la evaluación deben estar basadas en un modelo de "risk assessment" (evaluación de riesgos), lo que implica que los esfuerzos en auditar y evaluar los procedimientos internos han de estar relacionados directamente con la prevención y detección de aquellos riesgos más característicos o típicos de la actividad propia de la empresa. ${ }^{42}$

En general, no se han dado indicaciones respecto a la forma o periodicidad en que se desarrollen tales prácticas, dejándolas nuevamente a discreción de la organización de la empresa. Con todo, se ha resaltado la importancia de incorporar una vertiente externa (principalmente por medio de la auditoría externa y otros mecanismos) que han adquirido progresiva relevancia especialmente en la prevención de ciertos fraudes financieros.

Otra de las exigencias al sistema de evaluación y monitoreo que se desprenden de las FSGO, y que es fácilmente vinculable con una de sus principales finalidades, es la de que disponga de métodos rápidos y efectivos de comunicación acerca de sus resultados a la dirección y administración de la empresa. Al respecto, se ha señalado que un sistema satisfactorio de monitoreo, auditoría y evaluación sería aquel que provea a la administración de la organización una información suficiente para que se determine por éstos si el programa de cumplimiento es generalmente efectivo en la prevención y detección de conductas delictivas. ${ }^{43}$

El grado de información que sea necesario obviamente dependerá de las características propias de la organización (como su pasado relativo al cumplimiento de la ley, el tipo de unidades funcionales que posee, clase de prácticas propias de ésta y el tipo de riesgo que posee la actividad la misma).

\section{d) Sistemas de reporte o denuncia interna.}

En cuarto lugar, desde estas directrices se ha venido exigiendo la incorporación a la práctica empresarial de una serie de obligaciones vinculadas a la existencia de sistemas de reporte en su interior:

"5. C. Disponer de, y dar publicidad a un sistema que pueda incluir mecanismos que permitan el anonimato y la confidencialidad, de tal manera que los representantes y los

\footnotetext{
${ }^{42}$ En este sentido se manifiesta NIETO MARTÍN, A., "Responsabilidad social, gobierno corporativo y autorregulación: sus influencias en el Derecho penal de la empresa", Política Criminal n 5 (2008), p. 10, para el cual la "metodología esencial" en la confección de un sistema de auto-organización es el conocido como risk assessment.

${ }^{43}$ Report of the ad hoc advisory group, cit. nota ${ }^{\circ} 22, \mathrm{p} 77$.
} 


\section{Polít. crim. Vol. 8, № 16 (Diciembre 2013), Art. 6, pp. 544 - 573. [http://www.politicacriminal.cl/Vol_08/n_16/Vol8N16A6.pdf]}

empleados de la organización puedan informar y buscar consejo en relación con conductas delictivas presentes o potenciales sin tener miedo a posibles consecuencias".

Continuando con el análisis de estas exigencias, vinculadas a la valoración de la efectividad de un programa de cumplimiento, desarrolladas por las FSGO y especialmente por el reporte del "Advisory Group" ya citado, se puede decir que uno de los aspectos más problemáticos que se pretenden resolver en la actualidad en el marco de la criminalidad de empresa, es el de la adopción de mecanismos adecuados para que la organización empresarial no facilite la comisión de delitos, lo que implica la adopción de correctivos vinculados a la dificultad de control propia de las organizaciones complejas.

En este sentido, se reconoce que la organización empresarial depende, en gran medida, para la corrección de potenciales o actuales conductas ilícitas ocurridas dentro de la empresa, de la información que pueda ser proporcionada por sus empleados y miembros. ${ }^{44}$ Con todo, los mecanismos que se adopten con el objeto de permitir el acceso a tal tipo de información deben contemplar ciertos elementos mínimos asociados a la efectividad de éstos mismos.

Una de las dificultades vinculadas al acceso de este tipo de información (como la sospecha de que se comete o que se cometerá un delito en el marco de la actividad de la empresa) viene dada porque tanto la experiencia como las investigaciones llevadas a cabo en este ámbito ponen en evidencia que los integrantes de la empresa no estarían dispuestos a entregar información (por el potencial riesgo que implica para su tranquilidad en el desempeño de sus funciones) si es que no se dan ciertas condiciones vinculadas a la confidencialidad y protección posterior luego de la entrega de la misma. ${ }^{45}$

Los requisitos de confidencialidad y anonimato del reporte deben ser compatibles, eso sí, con las acciones que debiera adoptar la empresa una vez conocido el reporte. Es decir, se debe tener siempre en cuenta dos aspectos fundamentales vinculados al uso del reporte. El primero, guarda relación con una cuestión interna, esto es, el anonimato del reporte debe permitir continuar con una investigación tendente a impedir definitivamente que la conducta ilícita se lleve a cabo o a remediarla, y por otro lado, desde una perspectiva externa, se deben tomar en cuenta ciertas obligaciones de brindar información existentes en la legislación aplicable a la actividad empresarial y a una eventual entrega de este reporte en caso de que la empresa se enfrente a un litigio penal.

En este sentido se puede observar el desarrollo que ha tenido en la práctica empresarial y legislativa de Norteamérica, el tratamiento y la protección de los "whistleblower", especialmente luego de los últimos escándalos empresariales (como el caso Enron o

\footnotetext{
${ }^{44}$ Report of the ad hoc advisory group, cit. nota $\mathrm{n}^{\circ} 22, \mathrm{p} 77$.

${ }^{45}$ Report of the ad hoc advisory group, cit. nota $\mathrm{n}^{\circ} 22, \mathrm{p} 77$. Al respecto, sostienen que tanto la experiencia como la investigación han demostrado que los integrantes de la empresa se muestran reacios a efectuar tales reportes (de denuncia) a menos que se asegure un alto nivel de confidencialidad, así como que no exista posibilidad alguna de que se tomen medidas en su contra si el reporte se efectúa de buena fe. Ver también GORDON, "Implementation", cit. nota n 12, p. 55, en relación al concepto de "non-retaliation".
} 
ARTAZA, Osvaldo. "Sistemas de prevención de delitos o programas de cumplimiento. Breve descripción de las reglas técnicas de gestión del riesgo empresarial y su utilidad en sede jurídico penal"

Worldcom $)^{46}$ y lo útiles que resultaron los testimonios prestados por parte de los empleados de las respectivas organizaciones empresariales para la resolución de estos casos. ${ }^{47}$

e) Adopción de mecanismos disciplinarios y de incentivos internos.

En seguida, y como quinto principio o criterio rector vinculado a los mínimos exigibles de un programa efectivo de cumplimiento, se encuentra la adopción de "mecanismos disciplinarios o de incentivos internos": ${ }^{48}$

"5.D. El programa ético y de cumplimiento deberá promoverse y sancionarse de manera coherente a lo largo y ancho de la organización mediante (A) incentivos apropiados para actuar de acuerdo con el programa ético y de cumplimiento; y (B) medidas disciplinarias adecuadas para prevenir o detectar la conducta delictiva".

El principal objetivo de este criterio rector se relaciona con el reforzamiento de la cultura organizacional tendente al cumplimiento de la ley mediante la presencia de consecuencias efectivas (tanto de incentivo como sancionatorias) que demuestren que el modelo no es solo un documento sin aplicación en la práctica cotidiana de la empresa. ${ }^{49}$

f) Reacción adecuada y actualización permanente del modelo.

En sexto lugar, y conectado con el punto anterior, específicamente con la reacción esperable de la empresa una vez que se ha detectado la conducta delictiva, se menciona como otro de los requisitos mínimos de un programa efectivo de cumplimiento el de respuesta adecuada a la ofensa y adopción de mecanismos tendentes a la prevención de futuras faltas (rededication):

“7. Tras la detección de la conducta delictiva, la organización deberá adoptar las medidas oportunas para responder de manera adecuada a la conducta delictiva y para prevenir ulteriores conductas delictivas similares, lo cual incluye tener que efectuar las modificaciones que sean necesarias en el programa ético y de cumplimiento".

“c. Al implementar el subapartado (b), la organización deberá evaluar periódicamente el riesgo de que se cometan conductas delictivas y deberá tomar las medidas apropiadas para diseñar, implementar o modificar cada uno de los requisitos

\footnotetext{
${ }^{46}$ Para una descripción general de esta serie de casos ver FRIEDRICHS D., "Enron et al.: Paradigmatic white collar crime cases for the new century", Critical Criminology, Vol. 12 (2004), pp. 114 y ss.

${ }^{47}$ Report of the ad hoc advisory group, cit. nota $\mathrm{n}^{\circ} 24$, p. 80. Para más detalles ver, entre otros, NIETO, La responsabilidad penal de las personas jurídicas, cit. nota $\mathrm{n}^{\circ} 3$, pp. 255 y ss. RODRÍGUEZ YAGÜE, C., "La protección de los Whistleblowers por el ordenamiento español: aspectos sustantivos y procesales", en: VV.AA., Fraude y Corrupción en el Derecho penal económico europeo. Eurodelitos de corrupción y fraude, (Coords. ARROYO ZAPATERO, L., NIETO MARTÍN, A.), Cuenca: Ed. Universidad de Castilla-La Mancha, 2006, pp. 447 y ss.; RAGUÉS, R., “Héroes o traidores? La protección de los informantes internos (whistleblowers) como estrategia político-criminal”, InDret, 3/2006, pp. 7 y ss.; GORDON, "Implementation", cit. nota ${ }^{\circ} 12$, pp. 54 y ss.

${ }^{48}$ Report of the ad hoc advisory group, cit. nota $\mathrm{n}^{\circ} 22$, p. 86. En un sentido similar, GORDON, "Implementation", cit. nota $\mathrm{n}^{\circ} 12$, p. 56.

${ }^{49}$ GRUNER/BROWN, “Organizational Justice”, cit. nota ${ }^{\circ} 10$, p. 751.
} 


\section{Polít. crim. Vol. 8, No 16 (Diciembre 2013), Art. 6, pp. 544 - 573. \\ [http://www.politicacriminal.cl/Vol_08/n_16/Vol8N16A6.pdf]}

establecidos en el subapartado (b) para reducir el riesgo de que se cometan conductas delictivas que se ha identificado a través de este procedimiento".

Para terminar con el análisis de estos principios organizativos, se hace necesario reparar en ciertos aspectos que deben ser tomados en cuenta para valorar positivamente la conducta de la empresa desde las exigencias en el diseño de su sistema de control interno. Para esto las FSGO, por ejemplo, dan algunos criterios para definir qué medidas específicas deben adoptar las organizaciones empresariales atendiendo a tres criterios diversos: (i) la costumbre empresarial o los estándares requeridos por una regulación gubernamental susceptibles de aplicación; (ii) el tamaño de la organización; (iii) conductas delictivas similares. ${ }^{50}$

En primer lugar, desde las FSGO se señala que la no incorporación de las costumbres o prácticas empresariales (propias del sector económico de la empresa), así como de los diversos estándares requeridos por la regulación gubernamental que estén destinados a ese tipo de actividad (sean susceptibles de aplicación) pesará en contra de considerar que el programa ético y de cumplimiento resulta verdaderamente efectivo.

En segundo lugar, se debe reparar en el tamaño de la organización, ya que la tendencia actual es a establecer un mayor nivel de exigencia para las grandes organizaciones, lo que se debe, a grandes rasgos, a las mayores posibilidades que éstas conllevan de facilitar la comisión de conductas delictivas, principalmente por la dificultad en la detección del individualmente responsable. Pero también por la mayor dispersión de la información y competencias decisorias vinculadas a los procesos empresariales potencialmente lesivos, que tendrán como consecuencia que la empresa debe invertir adecuadamente en contrarrestar estos mismos factores de riesgo.

La diferencia con las pequeñas organizaciones no estaría dada en forma cualitativa (para ambos tipos de organizaciones se exige la misma eficacia y el mismo nivel de calidad de los mecanismos implementados), sino que se entiende desde una perspectiva cuantitativa, ya que a la pequeña organización empresarial no se le exigiría el mismo nivel de gasto en la implementación de estas medidas, así como el mismo nivel de formalidad que le son exigibles a las grandes empresas. ${ }^{51}$

En tercer lugar, se debe tener en cuenta que a la hora de definir la efectividad de un programa de cumplimiento habrá que considerar si la conducta delictiva es una reiteración de la misma empresa, lo que implicaría que ésta no actualizó adecuadamente los procedimientos internos adoptados, infringiendo uno de los criterios básicos de efectividad

\footnotetext{
${ }^{50}$ GÓMEZ-JARA, La responsabilidad penal, cit. nota ${ }^{\circ} 12$, pp. 127 y ss.

51 "Para cumplir con los requisitos de esta directriz, las pequeñas organizaciones deberán mostrar el mismo grado de compromiso con la conducta ética y con el cumplimiento con el Derecho que las Grandes organizaciones. No obstante, la pequeña organización puede cumplir con los requisitos de esta directriz con menos formalidad y menos recursos de lo que se espera de una gran organización. Si las circunstancias son las adecuadas, la confianza en los recursos existentes y los sistemas sencillos pueden mostrar un grado de compromiso que sólo podría demostrarse en una organización mediante unos sistemas planificados e implementados de manera más formal”, traducción de GÓMEZ-JARA, La responsabilidad penal, cit. nota ${ }^{\circ}$ 12 , p. 128.
} 
ARTAZA, Osvaldo. "Sistemas de prevención de delitos o programas de cumplimiento. Breve descripción de las reglas técnicas de gestión del riesgo empresarial y su utilidad en sede jurídico penal"

ya descritos. La conducta anterior de la empresa sería especialmente relevante a la hora de determinar cómo se configura su ámbito de responsabilidad, que en gran medida vendrá dado por la previsibilidad de riesgos que puedan derivarse de su funcionamiento, por lo que se entiende que conductas que hayan ocurrido con anterioridad tendrán un tratamiento diverso en esta sede de constatación de previsibilidad.

\section{Las reglas técnicas de gestión del riesgo, o de control interno, y su incidencia en el problema planteado.}

Como conclusión, se atenderá en forma breve a un caso ficticio con el objeto de presentar una primera aproximación relativa a cómo las reglas anteriormente estudiadas pueden ser de utilidad para la concreción del deber dirigido a la empresa de gestionar adecuadamente sus riesgos penales contenido en el art. 3 de la Ley 20.393 y concretado en el art. 4 del mismo cuerpo legal:

Una empresa productora $X$, debe obtener un permiso de la Administración Pública para poder operar en la localidad Y. Este permiso no podía ser obtenido en forma regular por el jefe de operaciones $Z$ encargado de la empresa $X$, ya que no contaban con todos los requisitos necesarios para tales efectos. Por lo mismo $\mathrm{Z}$ decide ofrecer un beneficio económico al funcionario público encargado de la concesión de tales permisos, lo que en definitiva permite que $\mathrm{Z}$ obtenga la autorización respectiva.

La conducta efectuada por $\mathrm{Z}$ podría ser enmarcada, sin mayores problemas, en la conducta típica descrita en el art. 250 de Código penal y, por ende, la persona jurídica (sociedad anónima) podría ser sujeto de atribución de responsabilidad penal si el delito (de cohecho) fue cometido directa e inmediatamente en su interés o para su provecho, por sus dueños, controladores, responsables, ejecutivos principales, representantes o quienes realicen actividades de administración y supervisión, por disposición expresa del art. $1^{\circ}$ y $3^{\circ}$ de la misma.

Se señaló al comienzo de este artículo, que la empresa no debía responder penalmente de todas las conductas delictivas cometidas por sus integrantes. Que en todo caso era necesario constatar algún nivel de conexión entre la misma y el hecho delictivo. En nuestro ordenamiento jurídico esto es evidente en la medida que el artículo $3^{\circ}$ de la Ley 20.393 el que exige tal conexión en forma expresa al señalar que la comisión del delito "fuere consecuencia del incumplimiento, por parte de ésta, de los deberes de dirección y supervisión".

Evidentemente estos deberes deben ser precisados, ya que no parecería adecuado que la empresa (al menos en sede jurídico-penal) respondiera en forma automática una vez se constata que el resultado lesivo deriva de su propia actividad. Este nivel no parece problemático en nuestro ámbito en donde en ningún caso se habría consagrado a nivel legislativo un sistema de transferencia de responsabilidad. La Ley 20.393 exige que el delito se haya debido (fuere consecuencia) de la omisión de tales deberes. 


\section{Polít. crim. Vol. 8, № 16 (Diciembre 2013), Art. 6, pp. 544 - 573. [http://www.politicacriminal.cl/Vol_08/n_16/Vol8N16A6.pdf]}

Con todo, la Ley chilena no solo se contenta con exigir tal conexión, sino que introduce también un filtro asociado a la idea de exigibilidad, como criterio general de delimitación de los deberes emanados del ordenamiento jurídico. Los deberes de supervisión y control no son absolutos, lo que sería imposible, sino que se satisfacen con la adopción e implementación, en forma anterior a la comisión del delito que se debiera haber prevenido, de un modelo de organización, administración y supervisión para prevenir delitos, o "modelos de prevención de delitos" de acuerdo a la terminología utilizada por el artículo $4^{\circ}$ de la Ley 20.393.

Esta disposición contiene una serie de medidas que debiera implementar la empresa para la prevención de ciertos delitos. Entre éstas destacan:

1) La designación, por parte de la máxima autoridad administrativa de la persona jurídica, de un encargado de prevención de delitos que cuente con los medios, facultades y la autonomía necesaria para ejercer su rol en forma eficiente, dentro de las que destacan el acceso directo a la administración de la empresa, con el objeto de comunicar a tiempo aquello que sea relevante para la prevención y detección oportuna de conductas delictivas, o la adopción de nuevas medidas en caso de que las existentes no parezcan suficiente.

2) El establecimiento (diseño e implementación) de un sistema de prevención de delitos, que deberá a) evaluar los riesgos penales propios de la empresa en concreto y; b) incluir los protocolos, reglas y procedimientos específicos que permitan a las personas que intervengan en las actividades o procesos evaluados como riesgosos, programar y ejecutar sus tareas o labores de una manera que prevenga la comisión de los delitos por los cuales se puede sancionar a la persona jurídica.

3) Por último, la Ley exige también que se especifiquen los medios que utilizará la empresa para administrar y auditar sus recursos financieros con el objeto de que no sean utilizados para la comisión de delitos, así como los procedimientos de denuncia interna y medidas disciplinarias (administrativas y pecuniarias) para quienes incumplan con lo dispuesto en el modelo de prevención de la empresa.

Como es evidente, el marco otorgado por el legislador es sumamente vago y genérico. Por ejemplo, ¿qué quiere decir que el encargado de prevención deba ser "autónomo"? o ¿cuándo se puede decir que cuenta con los medios y facultades suficientes para el cumplimiento de su rol? ¿Cuándo se entiende que cuenta con acceso directo a la administración?

Esta indeterminación se refleja especialmente en lo que respecta al diseño e implementación del sistema de prevención. ¿Qué metodología se exigirá a las empresas para la identificación y posterior evaluación de sus actividades riesgosas? ¿Cuándo se entenderá que el sistema responde adecuadamente a los riesgos propios de su actividad y no a otros? ¿Qué nivel de exigencia debieran contemplar los protocolos para la prevención de delitos específicos para satisfacer el deber de prevención de la empresa?, etc. ${ }^{52}$

\footnotetext{
${ }^{52}$ Un aspecto que no pudo ser abordado en esta oportunidad es el del papel que la Ley 20.393 le asigna a la certificación de los modelos de prevención de delitos y la consecuencia en sede procesal de la certificación del modelo. Sin embargo, parece evidente que las instituciones certificadoras debieran jugar un rol
} 
ARTAZA, Osvaldo. "Sistemas de prevención de delitos o programas de cumplimiento. Breve descripción de las reglas técnicas de gestión del riesgo empresarial y su utilidad en sede jurídico penal"

Por lo mismo, parece del todo razonable remitirse en este ámbito a la propia práctica del sector empresarial, con el objeto de detectar aquello que se exige a las empresas en relación a la gestión de sus propios riesgos. Una primera aproximación al problema nos conduce inmediatamente a detectar una serie de reglas técnicas asociadas a la prevención general de riesgos que pueden servir para empezar a dar respuesta a este nuevo problema al que se enfrenta la dogmática penal.

En primer lugar, se pudo observar como desde el mismo sector estudiado se reconoce que en la gestión de los propios riesgos de la empresa es fundamental el fomento de una cultura organizacional tendente al cumplimiento de la ley. Esto no es más que la contrapartida a ciertos efectos que eventualmente puede generar la misma actividad que derechamente facilitarían la inobservancia de la normativa vigente. Es decir, el funcionamiento diario de la empresa muchas veces puede ir generando ciertas prácticas que incidan en que sus integrantes pretendan cumplir los objetivos corporativos aún en contra de lo establecido por el ordenamiento jurídico.

En el caso propuesto más arriba, la conducta efectuada por Z, consistente en sobornar a un funcionario público, puede haber estado influida por ciertas dinámicas dadas al interior de la empresa, por ejemplo, debido a las políticas de obtención de bonos por resultados, o a las presiones que se ejercieron en la persona de $\mathrm{Z}$ a causa de los bajos rendimientos que había presentado en el último tiempo, por lo cual se le exigía una respuesta efectiva a tal situación bajo amenaza de ver mermada su situación en la organización. Incluso simplemente porque Z sabía que si obtenía la autorización, tal hecho sería mirado con buenos ojos por la alta dirección y que, por lo mismo, lo considerarían a los efectos de la promoción interna de la empresa.

Por ende, parece razonable que sea la empresa misma la que en el diseño de su actividad genere las respuestas adecuadas para contrarrestar esta eventual tendencia en sus integrantes. Por ejemplo, enviando un mensaje contundente a éstos, relativo a que los objetivos corporativos solo pueden alcanzarse en forma tal que se observe en todo momento el ordenamiento jurídico. La medida en que la empresa deba contrarrestar estas señales ambiguas de cara al cumplimiento de la ley, que pueda generar con las dinámicas internas, debe ser establecida observando las reglas técnicas descritas con anterioridad.

Así, por ejemplo, mediante la difusión de un código de conducta, y mediante los programas y procedimientos de capacitación y entrenamiento, tendentes a reforzar una cultura organizacional correcta, así como un conocimiento adecuado del orden jurídico por parte de los integrantes de la empresa.

protagónico en la delimitación de los niveles de cuidado que se le puede exigir a la empresa en el diseño del modelo, para lo cual deberían atender, principalmente a las reglas técnicas del sector en particular. Lo único que establece la Ley es que las certificadoras deberán dar cuenta acerca del cumplimiento de las exigencias contenidas en los números 1), 2) y 3) del artículo $4^{\circ}$ de la misma ley, por lo que el problema de la concreción señalado no se resuelve sino que más bien se traslada a expertos externos a la institución, sin perjuicio de que son los tribunales quienes en definitiva resolverán acerca del cumplimiento de este deber. 
Esto en lo que respecta al primer nivel de gestión del riesgo abordado. Sin embargo, se señaló que atender exclusivamente al aspecto valórico de la organización era insuficiente, siendo además necesario generar ciertas condiciones estructurales que permitieran prevenir en mejor forma eventuales conductas delictivas. Así, en el ejemplo propuesto, $\mathrm{Z}$ al decidir que otorgará al funcionario de la Administración una suma de dinero para la obtención del permiso debe enfrentarse al problema relativo a la obtención de tal suma. Para esto utiliza una parte de los recursos destinados a programas de responsabilidad social y donaciones (cuya utilización dependen del gerente respectivo, quien confía en el criterio de sus subalternos y, por ende, autoriza el gasto sin mayor problema cuando le señala que será para la compra de equipo para una escuela pública de la localidad respectiva). Una vez que se debe rendir el gasto, $\mathrm{Z}$ presenta en la Unidad de contabilidad el comprobante de autorización del gerente y nada más.

Si la empresa en cuestión permite rendición de gastos de la forma recién descrita es muy probable que esté facilitando, esta vez mediante su propia estructura y organización, la comisión de conductas delictivas en el sentido de poner a disposición del integrante todos los medios para la ejecución del delito. Una vez más, parece razonable que sea la misma empresa la que genere las medidas de cuidado para contrarrestar tales factores que facilitan la realización de conductas delictivas. En este caso, mediante la implementación de un sistema de control del gasto que responda a ciertos parámetros mínimos de diligencia.

A partir del análisis de las reglas técnicas relativas a la gestión del riesgo se pudo observar una serie de principios organizativos tendentes a asegurar un nivel mínimo de diligencia en el diseño organizacional de la empresa. Así, se mencionaron entre otros la importancia de que la empresa pueda detectar adecuadamente tal práctica como una actividad riesgosa, esto es, que establezcan mecanismos de evaluación de riesgos que le permita adoptar aquellas medidas que respondan a sus propios riesgos. Una vez que la empresa ha detectado sus actividades peligrosas no solo se deben definir aquellos mecanismos para contrarrestar o controlar las mismas, sino que principalmente las medidas de control para asegurar que tales procedimientos o protocolos se cumplan en forma efectiva. Dentro de éstos destacan ciertas exigencias relativas a la supervisión del sistema que se implemente por parte de la empresa y a las reglas de delegación de competencias en aquellas áreas que sean especialmente relevantes, así como las posibilidades de acceder oportunamente a información clave para poder detectar y reaccionar a tiempo frente a conductas inapropiadas.

A través de este ejemplo solo se quiere dar cuenta de estos dos niveles diversos que deben ser considerados para la prevención de las conductas delictivas cometidas en el marco de la actividad de la empresa. De esta manera se puede ir delimitando en mejor forma el ámbito de competencia que recae en la propia empresa en relación al deber que emana del ordenamiento jurídico de prevenir cierta gama de conductas delictivas que emanen del desarrollo de su propia actividad. 
ARTAZA, Osvaldo. "Sistemas de prevención de delitos o programas de cumplimiento. Breve descripción de las reglas técnicas de gestión del riesgo empresarial

y su utilidad en sede jurídico penal"

\section{BIBLIOGRAFÍA}

ALCÁCER GUIRAO, R., "Prevención y Garantías, conflicto y síntesis", DOXA n 25 (2002).

AMARELLI, G., "I criteri oggettivi di ascrizione del reato all'ente colectivo ed i reati in materia di sicurezza sul lavoro. Dalla teorica incompatibilità alla forzata convivenza", Diritto Penale Contemporaneo, 2013. Disponible en http://www.penalecontemporaneo.it/materia/-/-/-/2238i_criteri_oggettivi_di_ascrizione_del_reato_all_ente_collettivo_ed_i_reati_in_mate ria_di_sicurezza_sul_lavoro/ (última visita 20/05/2013).

ARTAZA VARELA, O., La empresa como sujeto de imputación de responsabilidad penal. Fundamentos y límites, Barcelona: Ed. Marcial Pons, 2013.

BACIGALUPO ZAPATER, E., Compliance y Derecho penal, España: Ed. Aranzadi, 2011.

DE SIMONE, G., "La responsabilità da reato degli enti: natura giuridica e criteri (oggettivi) di imputazione", Diritto Penale Contemporaneo, 2012. Disponible en http://www.penalecontemporaneo.it/materia/-/-/-/1809-

la_responsabilit da_reato_degli_enti natura_giuridica_e_criteri_oggettivi_di_i mputazione/ (última visita 20/05/2013).

ETHICS RESOURCE CENTER, Leading Corporate Integrity: Defining the Role of the Chief Ethics and Compliance Officer, 2007. Disponible en http://www.ethics.org/files/u5/RPceco.pdf (última visita 20/05/2013).

FEIJOO SÁNCHEZ, B., Tratado de responsabilidad penal de las personas jurídicas (BAJO FERNÁNDEZ, M., FEIJOO SÁNCHEZ, B., GÓMEZ-JARA DIEZ, C.), España: Ed. Civitas/Thomson Reuters, 2012.

FOFFANI, L., "Escándalos económicos y reformas penales: Prevención y represión de las infracciones societarias en la era de la globalización", $R P \mathrm{n}^{\circ} 23$ (2009).

FRIEDRICHS D., "Enron et al.: Paradigmatic white collar crime cases for the new century", Critical Criminology, Vol. 12, 2004.

GÓMEZ-JARA DÍEZ, C., La responsabilidad penal de las Empresas en los EEUU, Sevilla: Ed. Fundación Ramón Areces, 2006.

GORDON, S., "Implementation of Effective Compliance and Ethics Programs and the Federal Sentencing Guidelines", en: VV. AA., Corporate Compliance Answer Book 2010, Estados Unidos: Ed. Practising Law Institute, 2010. Capítulo 2 disponible en http://www.pli.edu/product_files/booksamples/26962_sample2.pdf (última visita 20/05/2013).

GRUNER R., Corporate Criminal Liability and Prevention, New York: Ed. Law Journal Press, 2010.

GRUNER R./BROWN L.,"Organizational Justice: Recognizing and Rewarding the Good Citizen Corporation", The Journal of Corporation Law, Vol. 21 (1996).

HECHLER BAER, M., "Governing Corporate Compliance”, Boston College Law Review, Vol. 50 (2009).

HEFENDEHL, R., "Enron, WorldCom, and the Consequences: Business Criminal Law Between Doctrinal Requirements and the Hopes of Crime Policy", Buf. Crim. L. Rev., Vol. 8 (2005). 
Polít. crim. Vol. 8, № 16 (Diciembre 2013), Art. 6, pp. 544 - 573.

[http://www.politicacriminal.cl/Vol_08/n_16/Vol8N16A6.pdf]

HERNÁNDEZ, BASUALTO H., "La introducción de la responsabilidad penal de las personas jurídicas en Chile", Política Criminal, Vol. 5, No 9 (Julio 2010), Art. 5, http://www.politicacriminal.cl/Vol_05/n_09/Vol5N9A5.pdf, (última visita 20/05/2013).

HUFF, K., "The role of corporate compliance programs in determining corporate criminal liability: A suggested approach”, Columbia Law Review, Vol. 96, 1996.

KINDHÄUSER, U., "El denominado riesgo no permitido", en: KINDHÄUSER, U.; POLAINO-ORTS, M.; BUOMPADRE, Jorge, Normativismo en Derecho penal. Estudios de dogmática jurídico-penal, Chaco: Ed. ConTexto, 2011.

LAUFER, W., "Corporate Liability, Risk Shifting, and the Paradox of Compliance", Vanderbilt Law Review, Vol. 52, 1999.

LOUGHMAN, B.; SIBERY, R., Bribery and Corruption. Navigating the Global Risks, Ed. Wiley Corporate F\&A, Kindle Edition.

Modello organizzativo, parte generale, de la empresa Fullsix Italia, 2013. Disponible en http://www.fullsix.it/wpcontent/uploads/2013/09/v04_modello_organizzativo_parte_generale_draft_01.pdf (última visita 20/05/13).

MOELLER, R., Coso Enterprise Risk. Establishing Effective Governance, Risk, and Compliance Processes, Ed. Wiley Corporate F\&A, (2 a edición), Kindle Edition.

MOLINA FERNÁNDEZ, F., "Presupuestos de la responsabilidad jurídica (análisis de la relación entre libertad y responsabilidad)", ADPCP, Vol. LIII (2000).

NIETO MARTÍN, A., "Responsabilidad social, gobierno corporativo y autorregulación: sus influencias en el Derecho penal de la empresa", Política Criminal no 5 (2008).

, La responsabilidad penal de las personas jurídicas: Un modelo legislativo, Madrid: Ed. Iustel, 2008.

NINO, C., Los escritos de Carlos s., en: NINO, Fundamentos de Derecho penal, Argentina: Ed. Gedisa, 2008.

RAGUÉS I VALLES, R., “¿Héroes o traidores? La protección de los informantes internos (whistleblowers) como estrategia político-criminal”, InDret, 3/2006.

Report of the ad hoc advisory group on the organizational sentencing guidelines, 2003. Disponible

en http://www.ussc.gov/Guidelines/Organizational_Guidelines/advgrprpt/advgrprpt.htm (última visita 20/05/2013).

RODRÍGUEZ YAGÜE, C., "La protección de los Whistleblowers por el ordenamiento español: aspectos sustantivos y procesales", en: VV.AA., Fraude y Corrupción en el Derecho penal económico europeo. Eurodelitos de corrupción y fraude, (Coords. ARROYO ZAPATERO, L., NIETO MARTÍN, A.), Cuenca: Ed. Universidad de Castilla-La Mancha, 2006.

PIÑA ROCHEFORT, J.I., Modelos de prevención de delitos en la empresa, Santiago: Ed. Abeledo Perrot/Thomson Reuters, 2012.

SALVADOR CODERCH, P.; GÓMEZ LIGÜERRE, C., "Respondeat Superior II. De la responsabilidad por hecho de otro a la responsabilidad de la organización”, en InDret, Julio 2002. Disponible en http://www.indret.com/pdf/088_es.pdf, (última visita 20/05/2013.

SCHÜNEMANN, B., "The Sarbanes-Oxley Act of 2002: A German Perspective", Buffalo Criminal Law Review, Vol. 8 (2004). 
ARTAZA, Osvaldo. "Sistemas de prevención de delitos o programas de cumplimiento. Breve descripción de las reglas técnicas de gestión del riesgo empresarial y su utilidad en sede jurídico penal"

SHARP PAINE, L., "Managing for Organizational Integrity", Harvard Business Review, Marzo-Abril, 1994.

SILVA SÁNCHEZ, J., Aproximación al Derecho penal contemporáneo, Montevideo: Ed. B de F, 2010 ( $2^{\text {a }}$ edición). 2003.

TOFFEL, M., “Industry Self-Regulation: What's Working (and What's Not)?”, para Harvard Business School, 2007. Disponible en Web: http://hbswk.hbs.edu/item/5590.html, (última visita 26/08/2013).

WALSH, C.; PYRICH, A., "Corporate Compliance Programs as a Defense to Criminal Liability: Can a Corporation Save its Soul?", Rutgers Law Review, Vol. 47 (19941995).

WELLNER P., "Effective Compliance Programs and Corporate Criminal Prosecutions", Cardozo Law Review, Vol. 27 (2005). 